This article was downloaded by: [University of California, Riverside]

On: 18 February 2010

Access details: Access Details: [subscription number 918975371]

Publisher Taylor \& Francis

Informa Ltd Registered in England and Wales Registered Number: 1072954 Registered office: Mortimer House, 3741 Mortimer Street, London W1T 3JH, UK

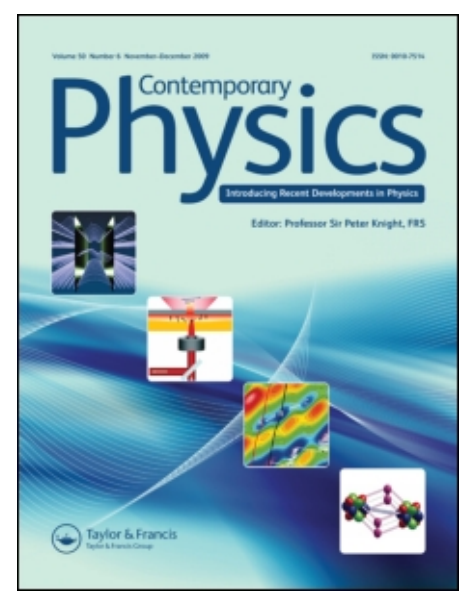

\title{
Contemporary Physics
}

Publication details, including instructions for authors and subscription information:

http://www.informaworld.com/smpp/title content=t713394025

\section{Uncollapsing the wavefunction by undoing quantum measurements}

Andrew N. Jordan a; Alexander N. Korotkov ${ }^{\mathrm{b}}$

${ }^{a}$ Department of Physics and Astronomy, University of Rochester, Rochester, New York, USA ${ }^{\mathrm{b}}$

Department of Electrical Engineering, University of California, Riverside, CA, USA

First published on: 12 February 2010

To cite this Article Jordan, Andrew N. and Korotkov, Alexander N.(2010) 'Uncollapsing the wavefunction by undoing quantum measurements', Contemporary Physics, 51: 2, 125 - 147, First published on: 12 February 2010 (iFirst)

To link to this Article: DOI: $10.1080 / 00107510903385292$

URL: http://dx.doi.org/10.1080/00107510903385292

\section{PLEASE SCROLL DOWN FOR ARTICLE}

Full terms and conditions of use: http://www.informaworld.com/terms-and-conditions-of-access.pdf

This article may be used for research, teaching and private study purposes. Any substantial or systematic reproduction, re-distribution, re-selling, loan or sub-licensing, systematic supply or distribution in any form to anyone is expressly forbidden.

The publisher does not give any warranty express or implied or make any representation that the contents will be complete or accurate or up to date. The accuracy of any instructions, formulae and drug doses should be independently verified with primary sources. The publisher shall not be liable for any loss, actions, claims, proceedings, demand or costs or damages whatsoever or howsoever caused arising directly or indirectly in connection with or arising out of the use of this material. 


\title{
Uncollapsing the wavefunction by undoing quantum measurements
}

\author{
Andrew N. Jordan ${ }^{\mathrm{a} *}$ and Alexander N. Korotkov ${ }^{\mathrm{b}}$ \\ ${ }^{a}$ Department of Physics and Astronomy, University of Rochester, Rochester, New York 14627, USA; ${ }^{b}$ Department of Electrical \\ Engineering, University of California, Riverside, CA 92521, USA
}

(Received 17 June 2009; final version received 2 October 2009)

\begin{abstract}
We review and expand on recent advances in theory and experiments concerning the problem of wavefunction uncollapse: given an unknown state that has been disturbed by a generalised measurement, restore the state to its initial configuration. We describe how this is probabilistically possible with a subsequent measurement that involves erasing the information extracted about the state in the first measurement. The general theory of abstract measurements is discussed, focusing on quantum information aspects of the problem, in addition to investigating a variety of specific physical situations and explicit measurement strategies. Several systems are considered in detail: the quantum double dot charge qubit measured by a quantum point contact (with and without Hamiltonian dynamics), the superconducting phase qubit monitored by a SQUID detector, and an arbitrary number of entangled charge qubits. Furthermore, uncollapse strategies for the quantum dot electron spin qubit, and the optical polarisation qubit are also reviewed. For each of these systems the physics of the continuous measurement process, the strategy required to ideally uncollapse the wavefunction, as well as the statistical features associated with the measurement are discussed. We also summarise the recent experimental realisation of two of these systems, the phase qubit and the polarisation qubit.
\end{abstract}

Keywords: wavefunction uncollapse; measurement reversal; quantum Bayesianism

\section{Introduction}

The irreversibility of quantum measurement is an axiomatic property of textbook quantum mechanics [1]. In his famous article Law without law, John Wheeler expresses the idea with poetic flare: 'We are dealing with [a quantum] event that makes itself known by an irreversible act of amplification, by an indelible record, an act of registration' [2]. However, it has been gradually recognised that the textbook treatment of an instantaneous wavefunction collapse is really a very special case of what is in general a dynamical process continuous quantum measurement. Continuous measurements do not project the system immediately into an eigenstate of the observable, but describe a process whereby the collapse happens over a period of time [3-13]. The fact that continuous measurement is a dynamical process with projective measurement as a special case, leads us to ask whether the irreversibility of quantum measurement is also a special case. The purpose of this paper is to review and expand on recent developments in this area of research, showing that it is possible to undo a quantum measurement, thereby uncollapsing the wavefunction, and to describe this physics in detail for both the abstract and concrete physical realisations.

This paper follows our earlier work on the subject $[14,15]$, as well as other papers investigating similar questions [16,17]. Wavefunction uncollapse teaches us several things about the fundamentals of quantum mechanics. First, there is a notion that wavefunction represents many possibilities, but that reality is created by measurement. The fact that the effects of measurement can be undone suggests that this idea is flawed, or at least too simplistic. If you create reality with quantum measurements, does undoing them erase the reality you created? Secondly, there is a wide-spread belief that quantum measurement is nothing more than a decoherence process. This suggests that the superposition never really collapses; it only appears to collapse. What actually happens, according to this idea, is that all the information about the system disperses into the environment: when a quantum system interacts with a classical measuring device, it becomes irreversibly entangled with all the particles that make up the measuring device and its surroundings. The uncollapse of the wavefunction demonstrates that decoherence theory cannot be the whole story, because a true decoherence process is irreversible [18]. The perspective we take in this paper further advances the 'quantum Bayesian' point of view, where the quantum state is nothing more than a reflection of our information about the system. When we receive more information about the system, the state changes or collapses not because

*Corresponding author. Email: jordan@pas.rochester.edu 
of any mysterious forces, but simply as a result of Bayesian updating.

We note that there are many approaches to the non-projective (continuous, weak, generalised, etc.) quantum measurement, which are essentially equivalent, in spite of quite different formalisms and terminology. To name just a few, let us mention mathematical POVM-type approaches [3,4] (POVM stands for positive operator-valued measure), method of restricted path integrals [5,6], analysis of quantum jumps in atomic shelving [7], quantum trajectories [8], quantum-state diffusion [9], Monte-Carlo wavefunction method [10], weak values [11], quantum filtering [12], and the Bayesian formalism for weak measurements in solid-state systems [13]. In this paper we will use the Bayesian and POVM-type formalisms because they are most suitable for the physical setups of our main interest.

Our work is indirectly related to the quantum eraser' of Scully and Drühl [19]. There, the which-path information of a particle is encoded in the quantum state of an atom, resulting in a destruction of interference fringe visibility. On the other hand, if the which-path information of the particle is erased, the interference fringes are restored. Both the Scully proposal and our proposal erase information. However, there is an important difference. In order for the uncollapsing procedure to work, we have to erase the information that was already extracted classically. In the 'quantum eraser', only potentially extractable information is erased.

Rather than begin with the abstract idea of uncollapse, we first introduce the concept with actual measurement processes in specific physical contexts. This brings to mind the saying of Asher Peres: 'Quantum phenomena do not occur in a Hilbert space. They occur in a laboratory' [20]. Following Peres' dictum, we discuss measurement processes in a variety of solid state systems, where there has been remarkable experimental progress in recent years. Quantum coherence has been demonstrated to occur in a controllable fashion in systems such as semiconductor quantum dots and superconducting Josephson junctions. We will discuss the physics of measurement in these systems, as well as concrete strategies for uncollapsing the wavefunction. It should be stressed that two of these proposals (a superconducting phase qubit and optical polarisation qubit) have now been implemented in the laboratory $[21,22]$, providing conclusive demonstration of wavefunction uncollapse. It is still too early to predict whether uncollapse can eventually become a really useful tool or it will remain only as a surprising and educating curiosity [18]. One possibly useful application is suppression of decoherence by the uncollapse procedure [23], which will be briefly discussed in our paper.

The paper is organised as follows. We introduce the general subject with a preliminary discussion in Section 2. We then give some specific examples in Sections 3 (the superconducting phase qubit) and 4 (the double quantum dot qubit, monitored by a quantum point contact). With these physical implementations, we discuss the uncollapsing strategies and results. In Section 5 we give a general treatment of the physics, using the POVM-based formalism. This is done both for pure states (Section 5.1) and mixed states (Section 5.2). In Section 5.3 we discuss the interpretation of wavefunction uncollapse, and what it tells us about quantum information. These general results are applied to the examples given previously in Section 5.4. In Section 6 we further apply the general results to the case of the finite-Hamiltonian qubit undergoing the uncollapse process. In Section 7, we generalise to the case of many charge qubits, and discuss an explicit procedure to undo any generalised measurement. We discuss recent developments in the theory and experiments of measurement reversal in Section 8 and conclude in Section 9. The Appendix contains results about the statistics of the waiting time distribution in the charge qubit example.

\section{Preliminary discussion}

Our goal is to restore an initial quantum state disturbed by measurement. However, it is important to discuss what exactly we mean by that. For example, if we start with a known pure state $\left|\psi_{\text {in }}\right\rangle$ and perform a textbook projective measurement, then it is trivial to restore the initial state: since we also know the postmeasurement wavefunction $\left|\psi_{m}\right\rangle$, we just need to apply a unitary operation which transfers $\left|\psi_{m}\right\rangle$ into $\left|\psi_{\text {in }}\right\rangle$. If we start with a known mixed state, then its restoration after a projective measurement is a little more involved $;^{1}$ however, such a procedure still can be easily analysed using standard quantum mechanics and classical probability theory.

In this paper we consider a different, non-trivial situation: we assume that an arbitrary initial state is unknown to us, and we still want to restore it after the measurement disturbance. To make this idea more precise, we consider a contest between the uncollapse proponent Plato, and an uncollapse skeptic Socrates. Socrates prepares a quantum system in any state he likes, but it is unknown to Plato. Socrates sends the state to Plato, who makes some measurement on the system, verified by the arbiter Aristotle. Plato then tries to undo the measurement. If Plato judges that the attempt succeeded, the system is returned to Socrates, with the claim that it is the original state. Socrates is 
then allowed to try and find a contradiction in any way he likes, with the whole process monitored by Aristotle. If a contradiction can be found, then he can claim to refute the uncollapse claim, but in the absence of contradiction, the uncollapse claim stands. If Socrates would like to try again to find a contradiction, or if Plato judges that his undoing attempt was unsuccessful (and does not return a state), then Socrates prepares a new (still unknown to Plato) state, and the competition continues. If Socrates cannot find a contradiction after many rounds of the competition, then Plato will win the contest, and will have successfully demonstrated the uncollapsing of the quantum state.

A slightly different but equivalent situation is when we know the initial state, but our uncollapsing procedure must be independent of the initial state (so we can pretend that it is unknown), and therefore the uncollapsing should restore any initial state in the same way. This formulation is most appropriate for a real experiment demonstrating the uncollapsing. Finally, we may consider the more general case where the measured system is entangled with another system, and we wish to restore the initial state of the compound system without any access to its second part.

The traditional statement of irreversibility of a quantum measurement can be traced to the fact that it may be described as a mathematical projection. Projection is a many-to-one mapping in the Hilbert space, and therefore the same post-measurement state generally corresponds to (infinitely) many initial states. $^{2}$ It is therefore impossible to undo a projective measurement.

However, the situation is different for a general [4] (POVM-type) measurement, which typically corresponds to a one-to-one mapping $\left|\psi_{\text {in }}\right\rangle \rightarrow\left|\psi_{m}\right\rangle$ in the Hilbert space of wavefunctions (in this paper we consider only 'ideal' measurements which do not introduce extra decoherence). In this case the postmeasurement wavefunction $\left|\psi_{m}\right\rangle$ can still be associated with the unique initial state $\left|\psi_{\text {in }}\right\rangle$, and a well-defined inverse mapping exists mathematically. This makes the uncollapsing possible in principle. Since the inverse mapping is typically non-unitary, it cannot be realised as an evolution with a suitable Hamiltonian. However, it can be realised using another POVM-type measurement with a specific ('lucky') result.

Rather than giving the most general, abstract case first, we start with a couple of specific examples of realistic systems where such a measurement either has been done, or could be done in the near future. Once the reader has the basic idea, we will proceed to generalise the process and develop its characteristics.

\section{Example 1: the phase qubit}

We first give a simple example of erasing information and uncollapsing the wavefunction for the case of a superconducting phase qubit [24]. The system is comprised of a superconducting loop interrupted by one Josephson junction (Figure 1(a)), which is controlled by an external flux $\phi_{e}$ in the loop. Two qubit states $|0\rangle$ and $|1\rangle$ (Figure $1(b)$ ) correspond to two lowest states in the quantum well for the potential energy $V(\phi)$, where $\phi$ is the superconducting phase difference across the junction. Transitions between the levels $|0\rangle$ and $|1\rangle$ (Rabi oscillations) can be induced by applying microwave pulses that are resonant with the energy level difference. Because of the energy difference, the two basis states acquire a phase difference between them, which linearly increases in time. However, this can be eliminated by going into the rotating frame; we always assume it in this section.

The qubit is measured by lowering the barrier (which depends on $\phi_{\mathrm{e}}$ ), so that the upper state $|1\rangle$ tunnels into the continuum with the rate $\Gamma$, while the state $|0\rangle$ does not tunnel out. The tunnelling event is sensed by a two-junction detector SQUID inductively coupled to the qubit (Figure 1(a)).

\subsection{Partial collapse}

For sufficiently long tunnelling time $t, \Gamma t \gg 1$, the measurement is a (partially destructive) projective measurement [24]: the system is destroyed if the tunnelling occurs, while if there is no record of tunnelling, then the state is projected onto the lower state $|0\rangle$. This measurement technique is remarkable in the fact that the wavefunction is collapsed if nothing happens. A more subtle situation arises if the barrier is raised after a finite time $t \sim \Gamma^{-1}$ [25]. The system is still destroyed if tunnelling happens, while in the case of no tunnelling (which we refer to as a null-result measurement) the state is partially collapsed because the inference from the measurement is ambiguous. This is due to the fact that

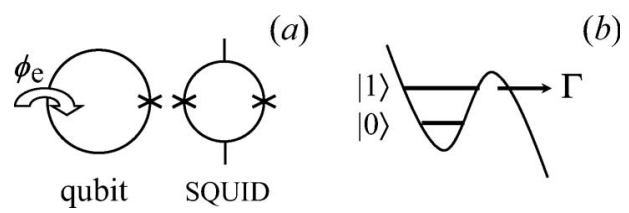

Figure 1. (a) Schematic of a phase qubit controlled by an external flux $\phi_{\mathrm{e}}$ and inductively coupled to the detector SQUID. (b) Energy profile $V(\phi)$ with quantised levels representing qubit states. The tunnelling event through the barrier is sensed by the SQUID. 
classically a null-result measurement could be because the system is in the lower energy state (and thus would not ever tunnel), or because it is in the upper energy level (and we simply didn't give it enough time).

The conditional probability for decaying if the qubit is in state $|1\rangle$ is given by $1-\exp (-\Gamma t)$. Given some initial density matrix $\rho_{\text {in }}$, we can interpret the diagonal density matrix elements $\left(\rho_{\text {in }, 00}\right.$ and $\left.\rho_{\text {in, }, 11}\right)$ as classical probabilities of being in states $|0\rangle$ or $|1\rangle$. We can then invoke the Bayes rule [26,27] to update the probabilities, ${ }^{3}$ given the data of "nothing happened'. It is not possible to use a simple general rule for updating the off-diagonal element $\rho_{01}$; however, analysis of the full dynamics in the extended Hilbert space [25,28] gives a simple result as well, so that the qubit density matrix changes after the nullresult measurement as

$$
\begin{gathered}
\rho_{00}(t)=\frac{\rho_{\mathrm{in}, 00}}{\rho_{\mathrm{in}, 00}+\rho_{\mathrm{in}, 11} \exp (-\Gamma t)}, \\
\rho_{11}(t)=\frac{\rho_{\mathrm{in}, 11} \exp (-\Gamma t)}{\rho_{\mathrm{in}, 00}+\rho_{\mathrm{in}, 11} \exp (-\Gamma t)}, \\
\rho_{01}(t)=\frac{\rho_{\mathrm{in}, 01} \exp [\mathrm{i} \varphi(t)] \exp (-\Gamma t / 2)}{\rho_{\mathrm{in}, 00}+\rho_{\mathrm{in}, 11} \exp (-\Gamma t)} .
\end{gathered}
$$

Such a measurement is ideal and does not decohere the qubit. Notice that in a simple model $[10,28]$ there is no relative phase $\varphi$ that is added during the measurement process, if one uses the rotating frame. In the real experiment [25], however, the energy difference between the states $|0\rangle$ and $|1\rangle$ changes in the process of measurement because it is affected by the changing external flux $\phi_{e}$, and therefore even in the (constantly) rotating frame the phase $\varphi$ is nonzero.

Actually, in the experiment [25] the situation is even more complex because the tunnelling rate gradually changes in time; also, instead of controlling the measurement time $t$, it is much easier to control the tunnelling rate. As a result, the measurement should be characterised by the overall strength $p_{t}=1-\exp \left[-\int_{0}^{t} \Gamma\left(t^{\prime}\right) \mathrm{d} t^{\prime}\right]$. Nevertheless, for simplicity, we use here the physically transparent language of Equations (1), (2) and (3) with $\exp (-\Gamma t)$ understood as $1-p_{t}$.

Up to such changes of notation, the coherent non-unitary evolution ((1), (2), (3)) has been experimentally verified in [25] using tomography of the postmeasurement state. The state tomography consisted of three types of rotations of the qubit Bloch sphere, followed by complete (projective) measurement. In the experiment it was not possible to select only the null-result cases, because it was not possible to distinguish if a tunnelling event happened during measurement or during tomography. However, a simple trick of comparing the protocols with and without tomography made it possible to separate the null-result cases.

\subsection{Uncollapsing}

We will now describe how to undo the state disturbance ((1), (2), (3)) caused by the partial collapse resulting from the null-result measurement. The undoing of this measurement consists of three steps [14]: (i) exchange the amplitudes for the states $|0\rangle$ and $|1\rangle$ by application of a microwave $\pi$-pulse, (ii) perform another measurement by lowering the barrier, identical to the first measurement, (iii) apply a second $\pi$-pulse. If the tunnelling did not happen during the second measurement, then the information about the initial qubit state is cancelled (both basis states have equal likelihood for two null-result measurements). Correspondingly, according to Equations (1), (2) and (3) (which are applied for the second time with exchanged indices $0 \leftrightarrow 1$ ), any initial qubit state is fully restored. An added benefit to this strategy is that the phase $\varphi$ is also cancelled automatically; the physics of this phase cancellation is the same as in the spin-echo technique for qubits.

It is easy to mistake the above pulse-sequence as simply the well known spin-echo technique alone. We stress that this is not the case: spin-echo deterministically reverses an unknown unitary transformation (arising, for example, from a slowly varying magnetic field) without gaining or losing any information about which state the system is in. Our strategy is probabilistic and requires erasing the classical information that one extracts from the system to begin with. It is a (probabilistic) reversal of a known non-unitary transformation - and therefore quite different from spin echo.

While both measurements should give null results for the success of our procedure, let us define the uncollapsing success probability $P_{\mathrm{S}}$ as the null-result probability of only the second measurement, assuming that the first measurement already gave the null result. Such a definition originates from our intention to characterise the probability of undoing the first measurement. (For the joint probability of two null results we will later use notation $\tilde{P}_{\mathrm{S}}$ ). To calculate $P_{\mathrm{S}}$, let us start with the initial qubit state $\rho_{\text {in }}$. Then after the first null-result measurement the qubit state is given by Equations (1), (2) and (3), and after the $\pi$-pulse the occupation of the upper level is $\tilde{\rho}_{11}=\rho_{\text {in }, 00} /$ $\left[\rho_{\text {in }, 00}+\rho_{\text {in }, 11} \exp (-\Gamma t)\right]$. The success probability is simply the probability that the second tunnelling 
will not occur, $P_{\mathrm{S}}=\tilde{\rho}_{00}+\tilde{\rho}_{11} \exp (-\Gamma t)=1-\tilde{\rho}_{11}(1-$ $\exp (-\Gamma t))$, which can be expressed as

$$
P_{\mathrm{S}}=\frac{e^{-\Gamma t}}{\rho_{\mathrm{in}, 00}+e^{-\Gamma t} \rho_{\mathrm{in}, 11}} .
$$

Notice that if $\Gamma t=0$ (i.e. the measurement has not started), then the success probability is 1 because the state never changed, and consequently does not need to be changed back. This is unsurprising. In the other limit, given a sufficiently long time of no tunnelling, $\Gamma t \gg 1$, the qubit state $|0\rangle$ is indicated with high confidence after the first null-result measurement, and consequently the uncollapse success probability becomes very small, recovering the traditional statement of irreversibility for a projective measurement. We stress that the possibility of uncollapsing as well as our formalism requires a quantum-limited detector, i.e. one that introduces no additional dephasing to the system. For such a detector measuring a pure state, the state remains pure throughout the partial collapse, and the uncollapse. We also note that if the qubit is entangled with other qubits, the uncollapsing restores the state of the whole system.

Uncollapsing of the phase qubit state has recently been experimentally realised by Nadav Katz and colleagues in the lab of John Martinis, at UC Santa Barbara [21]. The experimental protocol was slightly shorter than that described above: it was missing the second $\pi$-pulse, so the uncollapsed state was actually the $\pi$-rotation of the initial state. Shortening of the protocol helped in decreasing the duration of the pulse sequence, which was about $45 \mathrm{~ns}$, including the state tomography. Since the qubit energy relaxation and dephasing times were significantly longer, $T_{1}=450 \mathrm{~ns}$ and $T_{2}^{*}=350 \mathrm{~ns}$, the simple theory described above was sufficiently accurate. The same trick as for the partial-collapse experiment [25] was used to separate tunnelling events during the first, second, and tomography measurements, because the detector SQUID was too slow to distinguish them directly.

The uncollapse procedure should restore any initial state. However, instead of examining all initial states to check this fact, it is sufficient to choose four initial states with linearly independent density matrices and use the linearity of quantum operations [4]. In the experiment [21] the uncollapse procedure was applied to the initial states $(|0\rangle+|1\rangle) / 2^{1 / 2},(|0\rangle-i|1\rangle) / 2^{1 / 2},|0\rangle$, and $|1\rangle$, and then the results were expressed via the language of the quantum process tomography [4] (QPT). ${ }^{4}$ The experimental [21] QPT fidelity of the uncollapsing procedure was above $70 \%$ for $p_{t}<0.6$. A significant decrease of the uncollapsing fidelity for larger measurement strength $p_{t}$, especially for $p_{t}>0.8$, was due to finite $T_{1}$ time and the fact that the null-result selection preferentially selects the cases with energy relaxation events, so that the procedure should no longer work well when $1-p_{t}$ becomes comparable to the probability of energy relaxation. (It is interesting that in some range of parameters, the same procedure preferentially selects the cases without energy relaxation events, thus effectively suppressing qubit decoherence [23] - see Section 8.3.)

Exact uncollapsing requires an ideal detection, which does not decohere a quantum state; Equations (1), (2) and (3) correspond to such an ideal detection. However, if various decoherence mechanisms are taken into account [28], then only imperfect uncollapsing is possible. The theory of imperfect uncollapsing is a subject of further research.

\section{Example 2: double-quantum-dot charge qubit}

The next example we consider is illustrated in Figure 2: a charge qubit made of a double-quantum-dot (DQD), populated by a single electron, which is measured continuously by a symmetric quantum point contact (QPC). This setup has been extensively studied in earlier papers, both theoretically [29] and experimentally [30]. In contrast to the previous example, we will denote the basis states of the DQD charge qubit as $|1\rangle$ and $|2\rangle$ (the electron being in one dot, or the other), to be consistent with previous papers on this setup. The measurement is characterised by the average currents $I_{1}$ and $I_{2}$ corresponding to the states $|1\rangle$ and $|2\rangle$, and by the shot noise spectral density $S_{I .}{ }^{5}$ We treat the additive detector shot noise as a Gaussian, white, stochastic process, and assume the detector is in the weakly responding regime, $|\Delta I| \ll I_{0}$, where $\Delta I=I_{1}-$ $I_{2}$ and $I_{0}=\left(I_{1}+I_{2}\right) / 2$, with QPC voltage much larger

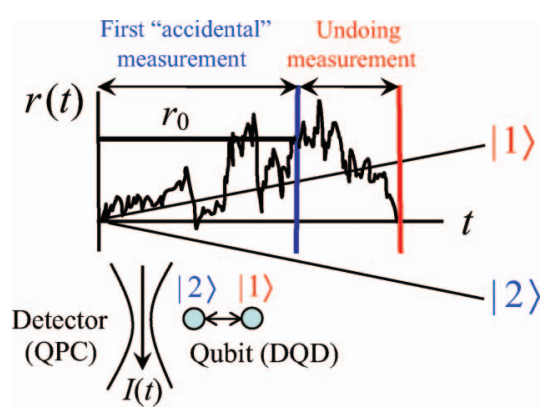

Figure 2. Illustration of the uncollapsing procedure for the charge qubit. The slanted lines indicate the deterministic output of the detector in the absence of noise, if the qubit is in state $|1\rangle$ or $|2\rangle$. The initial measurement yields the result $r_{0}$. The detector is again turned on, hoping that at some future time the measurement result $r(t)=r_{0}+r_{u}(t)$ crosses the origin, at which time the detector is turned off, successfully erasing the information obtained in the first measurement, and restoring the initial qubit state. 
than all other energy scales, so that the measurement process can be described by the quantum Bayesian formalism [13].

\subsection{Measurement dynamics for a non-evolving qubit}

We begin for simplicity with the assumption that there is no qubit Hamiltonian evolution, so that the qubit state evolves due to the measurement only (this can also be effectively done using 'kicked' quantum nondemolition (QND) measurements [31]). As was shown in [13], at low temperature the QPC is an ideal quantum detector (which does not decohere the measured qubit), so that the evolution of the qubit density matrix $\rho$ due to continuous measurement preserves the 'murity' [32] $\mathcal{M} \equiv \rho_{12} /\left(\rho_{11} \rho_{22}\right)^{1 / 2}$ while the diagonal matrix elements evolve according to the classical Bayes rule $[26,27] .{ }^{3}$ We define the electrical current through the QPC averaged in a time $t$ as $\bar{I}(t)=\left[\int_{0}^{t} I\left(t^{\prime}\right) \mathrm{d} t^{\prime}\right] / t$, and the quantum Bayesian equations read [13]

$$
\begin{aligned}
\rho_{11}(t) & =\frac{\rho_{11}(0) P_{1}(\bar{I})}{\rho_{11}(0) P_{1}(\bar{I})+\rho_{22}(0) P_{2}(\bar{I})}, \\
\rho_{22}(t) & =\frac{\rho_{22}(0) P_{2}(\bar{I})}{\rho_{11}(0) P_{1}(\bar{I})+\rho_{22}(0) P_{2}(\bar{I})}, \\
\mathcal{M} & =\rho_{12} /\left(\rho_{11} \rho_{22}\right)^{1 / 2}=\mathrm{const},
\end{aligned}
$$

where the conditional (Gaussian) probability densities of a current $\bar{I}$ realisation, given that the qubit is in $|1\rangle$, $|2\rangle$ are

$$
\begin{aligned}
P_{1,2}(\bar{I})= & \left(t / \pi S_{I}\right)^{1 / 2} \\
& \times \exp \left[-\left(\bar{I}-I_{1,2}\right)^{2} t / S_{I}\right] .
\end{aligned}
$$

Equations (5) and (6) may be simplified by noting

$$
\frac{\rho_{11}(t)}{\rho_{22}(t)}=\frac{\rho_{11}(0)}{\rho_{22}(0)} \exp [2 r(t)]
$$

where we define the dimensionless measurement result as

$$
\begin{aligned}
r(t) & =\frac{t \Delta I}{S_{I}}\left[\bar{I}(t)-I_{0}\right] \\
& =\frac{\Delta I}{S_{I}} \int_{0}^{t}\left[I\left(t^{\prime}\right)-I_{0}\right] \mathrm{d} t^{\prime} .
\end{aligned}
$$

Notice that $r(t)$ is closely related to the total charge passed through the QPC, and therefore $r(t)$ accumulates in time. For times much longer than the 'measurement time' [33] $T_{M}=2 S_{I} /(\Delta I)^{2}$ (the time scale required to obtain a signal-to-noise ratio of 1 ), the average current $\bar{I}$ tends to either $I_{1}$ or $I_{2}$ because the probability density $P(\bar{I})$ of a particular $\bar{I}$ is

$$
P(\bar{I})=\sum_{i=1,2} \rho_{i i}(0) P_{i}(\bar{I}),
$$

and $P_{i}(\bar{I}) \rightarrow \delta\left(\bar{I}-I_{i}\right)$ for $t / T_{M} \rightarrow \infty$ Therefore, $r(t)$ tends to $\pm \infty$, continuously collapsing the state to either $|1\rangle$ (for $r \rightarrow \infty$ ) or $|2\rangle$ (for $r \rightarrow-\infty$ ). Importantly, for the special case when the initial state is pure, the state remains pure during the entire process. Notice that the density matrix Equations (5), (6) and (7) formally coincide with Equations (1), (2) and (3) in the phase qubit example, if the qubit states are renumbered, the phase $\varphi$ is neglected, and $\Gamma t$ is replaced with $2 r$.

\subsection{Uncollapsing for the charge qubit}

In order to describe how to uncollapse the charge qubit state, we note that if $r(t)=0$ at some moment $t$, then the qubit state becomes exactly the same as it was initially, $\rho(t)=\rho(0)$, as follows from Equations (9) and (7). This of course must be the case if $t=0$, i.e. before the measurement began, but is equally valid for some later time. To see why this is so from the informational point of view, we note that in the absence of noise, the measurement result from states $|1\rangle,|2\rangle$ would simply be $r_{1,2}(t)= \pm t / T_{M}$. With the noise present, the measurement outcome $r(t)=0$ splits in half the difference between states $|1\rangle$ and $|2\rangle$. Such an outcome corresponds to an equal statistical likelihood of the states $|1\rangle$ and $|2\rangle$, and therefore provides no information about the state of the qubit.

Suppose the outcome of a measurement is $r_{0}$, partially collapsing the qubit state toward either state $|1\rangle$ (if $r_{0}>0$ ), or state $|2\rangle$ (if $r_{0}<0$ ). The previous 'no information' observation suggests the following strategy for uncollapsing [14]: continue measuring, with the hope that after some time $t$ the stochastic result of the second measurement $r_{\mathrm{u}}(t)$ becomes equal to $-r_{0}$, so the total result $r(t)=r_{0}+r_{\mathrm{u}}(t)$ is zero, and therefore the initial qubit state is fully restored. If this happens, the measuring device is immediately switched off and the uncollapsing procedure is successful (Figure 2). However, $r(t)$ may never cross the origin, and then the uncollapsing attempt fails. The probability of success $P_{\mathrm{S}}$ is therefore the probability that $r(t)$ crosses the origin (at least once) after it starts from $r_{0}$.

This strategy requires the observation of a particular measurement result that may never materialise. The strategy shifts the randomness to the amount of time that needs to elapse in order to find the desired measurement result. Of course, in a given realisation the measurement result could take on the desired value multiple times, so we will take as our strategy to turn off the detector the first time the measurement result 
takes on $r=0$. In the classical stochastic physics this is known as a first passage process [34], the theory of which is well developed and is detailed in Appendix 1.

In order to analyse the uncollapsing strategy performance, in particular to find the success probability $P_{\mathrm{S}}$, it is important to notice that the offdiagonal elements of the qubit density matrix $\rho$ do not come into play when we consider the detector output $I(t)$ (this is true only in the case of zero or QNDeliminated qubit Hamiltonian; in Section 6 we will generalise to the finite qubit Hamiltonian case). As a result, the quantum problem can be exactly reduced to a classical problem by substituting $\rho_{11}(t)$ and $\rho_{22}(t)$ with classical probabilities, evolving in the course of measurement according to the classical Bayes rule, while evolution of the off-diagonal elements $\rho_{12}=\rho_{21}^{*}$ can be found automatically from the murity conservation law (7). We therefore model the qubit by a classical bit with probability $p_{1}=\rho_{11}(0)$ of being prepared in state ' 1 ' and probability $p_{2}=\rho_{22}(0)$ of being in state ' 2 '. If the bit is in state ' 1 ', the dimensionless measurement result $r(t)$ evolves as a random walk with diffusion coefficient $D=(\Delta I)^{2} /$ $4 S_{I}=1 / 2 T_{M}$ and drift velocity $v_{1}=(\Delta I)^{2} / 2 S_{I}=$ $1 / T_{M}$ (see Equations (8) and (10)). For the bit state ' 2 ' the random walk of $r(t)$ has the same diffusion coefficient but the opposite drift velocity $v_{2}=-(\Delta I)^{2} /$ $2 S_{I}$. We are given the fact that the first part of the measurement had the result $r_{0}$ (i.e. we select only such realisations). We need to analyse the stochastic behaviour of the total measurement result $r(t)$ during the second part of measurement, with most attention to the crossing of the zero line $r(t)=0$ (for convenience we shift $t=0$ to the beginning of the second measurement, so that $\left.r(0)=r_{0}\right)$.

Let us find the probability $P_{\mathrm{S}}$ of such a crossing. Here we obtain it in a simple way, while in Appendix 1 we reproduce the result in a more complicated way, which also allows us to analyse the statistics of the waiting time. For definiteness take $r_{0}>0$ (this will be extended later). Then the result $r(t)$ will necessarily cross 0 if the bit is actually in the state ' 2 ', because in this case $r(t=\infty)=-\infty$ while $r(t=0)=r_{0}>0$. The probability of being in the state ' 2 ' is $\tilde{p}_{2}=p_{2} \exp$ $\left(-r_{0}\right) /\left(p_{1} \exp \left(r_{0}\right)+p_{2} \exp \left(-r_{0}\right)\right)$ from (9), which differs from $p_{2}$ because of the Bayesian update. ${ }^{3}$ If the bit is in state ' 1 ' (this happens with probability $\left.\tilde{p}_{1}=1-\tilde{p}_{2}\right)$, then $r(t=\infty)=+\infty$ and the crossing of 0 may never happen; however, it is still possible with some probability $P_{\mathrm{C}}$, which depends on $r_{0}$, and also on $D$ and $v_{1}$. To find $P_{\mathrm{C}}$, let us consider an infinitesimal time step $\mathrm{d} t$ and model the diffusion by discrete jumps in $r$ of magnitude $\Delta r= \pm(2 D \mathrm{~d} t)^{1 / 2}$. After a step $\mathrm{d} t$, the coordinate will then shift to one of two positions, $r=r_{ \pm}$, where $r_{ \pm}=r_{0}+v_{1} \mathrm{~d} t \pm(2 D \mathrm{~d} t)^{1 / 2}$. Each of these new coordinates will have its own probability of eventually crossing the origin, $P_{\mathrm{C}}\left(r_{ \pm}\right)$. Because the diffusive dynamics is generated by choosing either $r_{+}$or $r_{-}$with equal weighting, it follows in the limit $\mathrm{d} t \rightarrow 0$ that

$$
P_{\mathrm{C}}\left(r_{0}\right)=\sum_{ \pm} \frac{1}{2} P_{\mathrm{C}}\left(r_{ \pm}\right)
$$

Expanding $P_{\mathrm{C}}\left(r_{ \pm}\right)$in this relation in a Taylor series, we find from the linear in $d t$ term that

$$
D \partial_{r_{0}}^{2} P_{\mathrm{C}}=-v_{1} \partial_{r_{0}} P_{\mathrm{C}}
$$

where $\partial_{r_{0}}$ and $\partial_{r_{0}}^{2}$ denote the first and second derivatives with respect to $r_{0}$. Taking into account that $P_{\mathrm{C}}=1$ for $r_{0}=0$ and $P_{\mathrm{C}}=0$ for $r_{0}=\infty$, the above differential equation may be easily solved to find $P_{\mathrm{C}}=\exp \left(-v_{1} r_{0} / D\right)=\exp \left(-2 r_{0}\right)$. Now collecting the probabilities of the zero line crossing for both bit states, we find

$$
\begin{aligned}
P_{\mathrm{S}}= & \tilde{p}_{1} P_{\mathrm{C}}+\tilde{p}_{2}=\exp \left(-r_{0}\right) \\
& /\left(p_{1} \exp \left(r_{0}\right)+p_{2} \exp \left(-r_{0}\right)\right) .
\end{aligned}
$$

The derivation for $r_{0}<0$ is similar and leads to the extra factor $\exp \left(2 r_{0}\right)$, so that the crossing probability in both cases can be written as $P_{\mathrm{S}}=\exp \left(-\left|r_{0}\right|\right) \mid$ $\left(p_{1} \exp \left(r_{0}\right)+p_{2} \exp \left(-r_{0}\right)\right)$.

Thus, using the trick of reducing the quantum dynamics to the classical problem, we have found the probability of successful uncollapsing for a DQD charge qubit with no Hamiltonian evolution [14]:

$$
P_{\mathrm{S}}=\frac{\exp \left(-\left|r_{0}\right|\right)}{\exp \left(r_{0}\right) \rho_{\text {in }, 11}+\exp \left(-r_{0}\right) \rho_{\text {in }, 22}},
$$

where $\rho_{\text {in }}$ characterises the qubit state before the first measurement.

This result formally corresponds to Equation (4) with substitution $\Gamma t \rightarrow 2 r_{0}$ (for positive $r_{0}$ ), and its physical meaning is also similar. When the first measurement result indicates either qubit state with good confidence $\left(\left|r_{0}\right| \gg 1\right)$, the probability of success $P_{\mathrm{S}}$ given by Equation (15) becomes very small, eventually becoming $P_{\mathrm{S}}=0$ for a projective measurement, realised for $r_{0}= \pm \infty$. In the other limit of $r_{0}=0$, the success probability is unity because no time needs to elapse - the state is already undisturbed.

While the above method allows us to find the success probability $P_{\mathrm{S}}$, there are other important characteristics of the uncollapsing strategy, which we have not discussed, such as the mean waiting time required to uncollapse the state. This and other characteristics of the strategy can be calculated through the 
more powerful methods of first-passage theory. This method and the results obtained from it are discussed in Appendix 1.

\section{General formalism}

The previous two examples were fairly straightforward to analyse because the unitary dynamics was effectively turned off, and only the measurement dynamics needed to be accounted for. In general the situation is more complicated. For example, if the DQD qubit had a finite Hamiltonian while the QPC was on, there would be both measurement dynamics and Hamiltonian dynamics. This situation leads us to discuss first the general formalism in its most abstract form before turning our attention to this more complicated example.

\subsection{Formalism for wavefunctions}

Let us first consider a pure initial state $\left|\psi_{\text {in }}\right\rangle$, and postpone a generalisation to mixed states until Section 5.2. In the formalism of a general (ideal) quantum measurement [4] which transfers pure states into pure states, the measurement with result $m$ is associated with the linear Kraus operator $M_{m}$, so that the probability of result $m$ is

$$
P_{m}\left(\left|\psi_{\text {in }}\right\rangle\right)=\| M_{m}\left|\psi_{\text {in }}\right\rangle \|^{2},
$$

where $\|.$.$\| denotes the norm of the state, and the$ (conditioned) state after measurement is

$$
\left|\psi_{m}\right\rangle=\frac{M_{m}\left|\psi_{\text {in }}\right\rangle}{\left[P_{m}\left(\left|\psi_{\text {in }}\right\rangle\right)\right]^{1 / 2}},
$$

where the denominator makes $\left|\psi_{m}\right\rangle$ properly normalised. (Very often people prefer to omit this denominator and work with non-normalised states; this makes the mapping linear.) The operators $E_{m}=\mathrm{M}_{m}^{\dagger} M_{m}$ (called POVM elements [4]) are Hermitian and positive semidefinite by construction; these operators must obey the completeness relation $\Sigma_{m} E_{m}=1$, which ensures that the total probability of all measurement results is unity. A measurement operator $M_{m}$ can always be written as

$$
M_{m}=U_{m} E_{m}^{1 / 2}
$$

where $U_{m}$ is a unitary operator (an important special case is when $M_{m}=E_{m}^{1 / 2}$; this corresponds to the 'quantum Bayes theorem' [6,35]).

Now let us discuss wavefunction uncollapse in this general and abstract context [14]. The state disturbance rule (17) is typically a nonunitary one-to-one map in the Hilbert space. To undo the measurement with known result $m$, we have to realise a physical process corresponding to the nonunitary inverse operator $M_{m}^{-1}$, multiplied by an arbitrary constant (which is not important because of the normalisation). This can be accomplished with another measurement, possibly together with unitary operations. As shown below, we can realise measurement undoing if the second measurement realises a Krauss operator of the form

$$
L=C U_{L} E_{m}^{-1 / 2} V_{L},
$$

where $U_{L}$ and $V_{L}$ are any unitary operators, and $C$ is an unspecified constant that will be discussed later. (The operator $L$ also has a decomposition of the form $U E^{1 / 2}$, but with a different POVM element E.) The uncollapse then consists of three steps: first, the unitary operator $V_{L}^{\dagger} U_{m}^{\dagger}$ is applied to reverse the unitary part of $M_{m}$ and prepare for the second measurement. Next the measurement operator $L$ is applied. Finally the unitary operator $U_{L}^{\dagger}$ is applied to reverse the remaining unitary part of $L$. We can now see the effect of the uncollapsing operation on the state $\left|\psi_{m}\right\rangle$ by applying Equation (17) and the unitaries to find

$$
\left|\psi_{f}\right\rangle=\frac{U_{L}^{\dagger} L V_{L}^{\dagger} U_{m}^{\dagger}\left|\psi_{m}\right\rangle}{\| U_{L}^{\dagger} L V_{L}^{\dagger} U_{m}^{\dagger}\left|\psi_{m}\right\rangle \|}=\left|\psi_{\text {in }}\right\rangle
$$

thus restoring the original state, because $U_{L}^{\dagger} L V_{L}^{\dagger}$ $U_{m}^{\dagger} M_{m}=C$, which is removed by the normalisation. (The phase of $C$ is not important, since it affects only the overall phase of the wavefunction.)

However, in order for the operator $L$ to be physically realisable, the operator $L^{\dagger} L$ must belong to another complete set of POVM elements, and therefore all its eigenvalues must not exceed unity (otherwise some states will be assigned probabilities that are above unity; notice that the eigenvalues are nonnegative automatically). Since $L^{\dagger} L=|C|^{2} V_{L}^{\dagger} E_{m}^{-1} V_{L}$, its eigenvalues are directly related to the eigenvalues $p_{i}^{(m)}$ of the operator $E_{m}$. Expressing $E_{m}=\sum_{i} p_{i}^{(m)}|i\rangle\langle i|$, where the eigenvectors $|i\rangle$ form an orthonormal basis, the eigenvectors of $L^{\dagger} L$ are obviously $V_{L}^{\dagger}|i\rangle$, and the corresponding eigenvalues are $|C|^{2} / p_{i}^{(m)}$. Since all these eigenvalues must not exceed 1 , we find the following inequality on $|C|^{2}$,

$$
|C|^{2} \leq \min _{i} p_{i}^{(m)}=\min P_{m},
$$

where $\min P_{m}$ is the probability of the result $m$, minimised over all possible states $\left|\psi_{\text {in }}\right\rangle$ in the Hilbert space. The equality of $\min P_{m}$ to $\min _{i} p_{i}^{(m)}$ follows from Equation (16).

In general, the uncollapse cannot be accomplished deterministically, since we rely on a measurement with 
a specific result, corresponding to the operator $L$. We can calculate the uncollapse success probability $P_{\mathrm{S}}$ from Equation (16), with $M_{m} \rightarrow L$ and $\left.\psi_{\text {in }}\right\rangle \rightarrow$ $V_{L}^{\dagger} U_{m}^{\dagger}\left|\psi_{m}\right\rangle$,

$$
\begin{aligned}
P_{\mathrm{S}} & =\| L V_{L}^{\dagger} U_{m}^{\dagger}\left|\psi_{m}\right\rangle \|^{2} \\
& =\left\|\frac{C\left|\psi_{\text {in }}\right\rangle}{\left.\| P_{m}\left(\left|\psi_{\text {in }}\right\rangle\right)\right]^{1 / 2}}\right\|^{2}=\frac{|C|^{2}}{P_{m}\left(\left|\psi_{\text {in }}\right\rangle\right)} .
\end{aligned}
$$

Now using the bound (21) for $|C|^{2}$, we find the bound for the success probability of uncollapsing after the first measurement with result $m$ [14]:

$$
P_{\mathrm{S}} \leq \frac{\min P_{m}}{P_{m}\left(\left|\psi_{\text {in }}\right\rangle\right)}
$$

where the denominator is the probability of the result $m$ for a given initial state, while the numerator is this probability minimised over all possible initial states.

The bound (23) is one of the most important results (notice a similar result in [16]) and deserves discussion. First, this bound is exact in the sense that it is achievable by an optimal uncollapsing procedure. This is because the uncollapsing operator with $|C|=$ $\left(\min P_{m}\right)^{1 / 2}$ is still a physically allowed operator. As we will see later, the upper bound (23) is achievable in real experimental setups (in particular, in the examples discussed in the two previous sections). However, nonoptimal uncollapsing procedures, especially involving a sequence of measurements, can lead to smaller success probabilities (an example of non-optimal uncollapsing has been discussed in [36]; another example will be discussed at the end of Section 6). An analysis of the procedures with an arbitrary sequence of measurements and unitary operations is similar to the above: the corresponding measurement and unitary operators should simply be multiplied.

Notice that the success probability (22) and the inequality (23) depends on the initial state, which is unknown to the person performing the uncollapsing (Plato, see description in Section 2). Therefore, the success probability $P_{\mathrm{S}}$ can be calculated by the man who knows what the initial state is (Socrates), while Plato can only estimate $P_{\mathrm{S}}$; for example, he can calculate the worst-case scenario (the minimum of $P_{\mathrm{S}}$ over the accessible Hilbert space) or can calculate the average of $P_{\mathrm{S}}$ over all possible initial states (this procedure will be discussed in the next subsection).

Recalling the fact that it is not possible to undo a fully collapsed state due to the nature of projective measurement, the uncollapsing probability $P_{\mathrm{S}}$ should decrease with increasing strength of the first measurement. Qualitatively, a stronger measurement is one that tends to a projection, as the uncertainty in the measurement decreases. Mathematically, this means that some eigenvalues of $E_{m}$ become closer to 0 . As a consequence, $\min P_{m}$ becomes smaller (see Equation (21)), therefore lowering the upper bound for $P_{\mathrm{S}}$. For a projective measurement $P_{\mathrm{S}}=\min P_{m}=0$, thus making the state uncollapse impossible.

It is interesting to discuss the case when the initial state $\left|\psi_{\text {in }}\right\rangle$ is known to belong to a certain subspace of the Hilbert space, and we therefore wish to restore states only in this subspace. In this case, the calculation of min $P_{m}$ should be limited to this subspace, which may increase the bound (23) for the success probability $P_{\mathrm{S}}$. A trivial example of such a situation is when the initial state $\left|\psi_{\text {in }}\right\rangle$ is known to Plato. Then it is not necessary to minimise $P_{m}$ over all possible initial states in Equation (23), because the set of possible states consists of only one (known) state, thus allowing uncollapsing with $100 \%$ probability. This is exactly the case discussed at the beginning of Section 2.

\subsection{Formalism for density matrices}

So far we have dealt only with pure states; however, it is very simple to generalise the uncollapsing formalism to include density matrices. In this case the initial density matrix $\rho_{\text {in }}$ is transformed by the first measurement into the state [4]

$$
\rho_{m}=\frac{M_{m} \rho_{\mathrm{in}} M_{m}^{\dagger}}{P_{m}}
$$

where the probability $P_{m}$ of the measurement result $m$ is

$$
P_{m}\left(\rho_{\text {in }}\right)=\operatorname{Tr}\left(M_{m}^{\dagger} M_{m} \rho_{\text {in }}\right) .
$$

Using the uncollapsing procedure previously discussed and using the same measurement operator $L$ given by Equation (19), we find that the uncollapsed state

$$
\rho_{f}=\frac{U_{L}^{\dagger} L V_{L}^{\dagger} U_{m}^{\dagger} \rho_{m} U_{m} V_{L}^{\dagger} L^{\dagger} U_{L}}{\operatorname{Tr}\left(L^{\dagger} L V_{L} U_{m}^{\dagger} \rho_{m} U_{m} V_{L}^{\dagger}\right)}=\rho_{\text {in }}
$$

coincides with the initial state. The uncollapsing success probability $P_{\mathrm{S}}$ is equal to the denominator in Equation (26), and satisfies the relation

$$
P_{\mathrm{S}}=|C|^{2} / P_{m}\left(\rho_{\text {in }}\right)
$$

(as in Equation (22)). The constant $|C|^{2}$ is still limited by the inequality (21), and therefore the probability of success has the upper bound [14]

$$
P_{\mathrm{S}} \leq \frac{\min P_{m}}{P_{m}\left(\rho_{\text {in }}\right)},
$$


which is the same as the bound (23), except for the new notation in the denominator, which reminds us of the possibly mixed initial state. The minimisation of $P_{m}$ in the numerator should now be performed over the space of all possible initial mixed states; however, the result obviously coincides with the minimisation over the pure states only. Similar to the case discussed in Section 5.1, the inequality (28) is the exact bound; it is achieved by an optimal uncollapsing procedure, which maximises $|C|$.

If the initial state is pure, then the formalism of this subsection is trivially equivalent to the formalism of Section 5.1. It becomes more general in the case when the 'actual' initial state is mixed; for example, this happens when the initial state has been in contact with an unmonitored environment or Socrates prepares a state by a blind random choice from a set of pure states. A more interesting case for the result (28) is when the measured system is entangled with another system, which does not evolve by itself. Then the formalism can be applied to the compound system; however, the measurement probability $P_{m}$ depends only on the reduced initial density matrix, traced over the entangled second part. Therefore, in the entangled bipartite case the uncollapsing procedure restores the state of the whole system, while the success probability $P_{\mathrm{S}}$ is given by Equation (28) with $\rho_{\text {in }}$ being the reduced density matrix.

Another advantage of Equation (28) in comparison with Equation (23) can be seen by referring back to the preliminary discussion with the contest between Plato and Socrates. In the derivation of both results the initial state is the 'actual' initial state, which is known to Socrates, but typically unknown to Plato. However, as we will prove below, Equation (28) can still be used by Plato in a somewhat different sense: with $\rho_{\text {in }}$ being understood as an averaged density matrix representing a distribution of possible initial states. In this case, Equation (28) gives the uncollapsing probability averaged over this distribution. For example, if Plato knew that Socrates' strategy is to prepare one of two possible (nonorthogonal) states $\left|\psi_{1}\right\rangle,\left|\psi_{2}\right\rangle$, with probabilities $\mathcal{P}$ and $1-\mathcal{P}$, then he could find the average uncollapsing probability in two ways. The first method is that he could simply average the uncollapsing probabilities of the two states (also taking into account the information acquired in the first measurement, see below). Alternatively, he could recall that the random state preparation described above is equivalent to considering the initial density matrix $\rho_{\text {in }}=\mathcal{P}\left|\psi_{1}\right\rangle\left\langle\psi_{1}\right|+$ $(1-\mathcal{P})\left|\psi_{2}\right\rangle\left\langle\psi_{2}\right|$, and then apply the result (28) to this density matrix. ${ }^{6}$ In this way, in the absence of any information, Plato could estimate his typical success rate by calculating (28) for a fully mixed state, invoking the principle of indifference [27].
In the general case the above statement, that both ways of computing the averaged uncollapsing probability are equivalent, can be proven both logically and explicitly. For the logical proof we notice that Plato's judgment of successful uncollapsing does not depend on whether or not Socrates knows the randomly picked state; therefore, the average probability of the cases judged to be successful should be the same in both situations (whether or not Socrates knows what the state is). Now let us also prove this statement explicitly, thus checking that our formalism is selfconsistent. Suppose the initial state is prepared by Socrates by choosing randomly from a set of initial states $\rho^{(k)}$ with probabilities $\mathcal{P}_{k}$ (the most natural case is when initial states are pure, $\rho^{(k)}=\left|\psi_{k}\right\rangle\left\langle\psi_{k}\right|$; however, this is not necessary). Then the bound for the average probability of uncollapsing success $P_{\mathrm{S}}^{(\mathrm{av})}$ is the average of the bounds (28):

$$
P_{\mathrm{S}}^{(\mathrm{av})} \leq \sum_{k} \frac{\min P_{m}}{P_{m}\left(\rho^{(k)}\right)} \mathcal{P}_{k}^{\prime} .
$$

Notice, however, that $\mathcal{P}_{k}^{\prime}$ is the posterior probability distribution given the result $m$, which is different from $\mathcal{P}_{k}$. We may now invoke the classical Bayes rule ${ }^{3}$ $[26,27]$ to relate the posterior $\mathcal{P}_{k}^{\prime}$ to the prior $\mathcal{P}_{k}$ and the conditional probability $P_{m}\left(\rho^{(k)}\right)$ to have result $m$ given state $k$, so that

$$
\mathcal{P}_{k}^{\prime}=\frac{P_{m}\left(\rho^{(k)}\right) \mathcal{P}_{k}}{\sum_{\tilde{k}} P_{m}\left(\rho^{(\tilde{k})}\right) \mathcal{P}_{\tilde{k}}} .
$$

Substituting Equation (30) into Equation (29) and using $\sum_{k} \mathcal{P}_{k}=1$ in the numerator, we obtain

$$
P_{\mathrm{S}}^{(\mathrm{av})} \leq \frac{\min P_{m}}{\sum_{k} P_{m}\left(\rho^{(k)}\right) \mathcal{P}_{k}}=\frac{\min P_{m}}{P_{m}\left(\rho^{(\mathrm{av})}\right)},
$$

where $\rho^{(\mathrm{a} v)}=\sum_{k} \rho^{(k)} \mathcal{P}_{k}$ is the averaged initial state. This ends the proof that Equation (28) can be used for an unknown initial state, with $\rho_{\text {in }}$ being understood as the average of all possible initial states.

\subsection{Uncollapsing probability, information, and an irreversibility measure}

We defined the success probability $P_{\mathrm{S}}$ as a probability to uncollapse the post-measurement state $\rho_{m}$. We now wish to start counting the overall success probability $\tilde{P}_{\mathrm{S}}$ from the time before the first measurement, so that $\tilde{P}_{\mathrm{S}}$ is the probability of the pair of events: measurement with result $m$ and then successful uncollapsing. Using Equations (27) and (28) we easily find the relation

$$
\tilde{P}_{\mathrm{S}}=P_{m}\left(\rho_{\text {in }}\right) P_{\mathrm{S}}=|C|^{2}
$$


and the upper bound

$$
\tilde{P}_{\mathrm{S}} \leq \min P_{m} .
$$

Notice that $\tilde{P}_{\mathrm{S}}$ is independent of the initial state. While this property may seem somewhat surprising, we will see later why it is rather obvious.

If we now wish to consider all possible results of the first measurement, and perform different uncollapsing procedures for each measurement result, then the total probability of uncollapsing $\tilde{P}_{\mathrm{S}}^{\text {total }}$ is bounded as

$$
\tilde{P}_{\mathrm{S}}^{\text {total }} \leq \sum_{m} \min P_{m}
$$

and is also independent of the initial state. The bounds (33) and (34) are exact and reachable by optimal uncollapsing procedures. The bound (34) indicates that $1-\Sigma_{m}$ min $P_{m}$ can be used as a measure of irreversibility (collapse strength) due to the measurement operation.

Now let us discuss the relationship between the uncollapsing procedure and our knowledge of the initial state. While uncollapsing is possible even if we know nothing about the initial state of the system, at first glance it seems like we gain some knowledge about the initial state in the process. This leads to the following interesting paradox, initially considered by Royer [37]. By doing both a measurement and unmeasurement, one can seemingly learn something about the initial state without disturbing it. Then by repeating the measurement + unmeasurement process many times, even though the probability of such an event rapidly decreases to zero, the successful event would lead to essentially perfect knowledge of the initial state, leaving the state itself perfectly intact! One could then violate a host of known results, such as the no-cloning theorem.

The resolution of the paradox lies in the fact that the pair of measurement and unmeasurement actually brings exactly zero information. Uncollapsing the state can only occur when the information in the second measurement exactly contradicts the information gained in the first measurement, thus nullifying it. This can happen in weak quantum measurements because there is uncertainty about the system in the measurement result. It is to the extent that this ambiguity exists that it is possible to undo the weak measurement. Let us examine this in more detail.

We learn something about a pre-measurement state when the measurement result depends on the state. The measurement with result $m$ brings some information about $\rho_{\text {in }}$ because the probability $P_{m}\left(\rho_{\text {in }}\right)$ depends on the initial state $\rho_{\text {in }}$. The ability to successfully uncollapse the state also brings some information about the initial state because the uncollapsing probability
$P_{\mathrm{S}}=|C|^{2} / P_{m}\left(\rho_{\text {in }}\right)$ also depends on $\rho_{\text {in }}$. However, the collapse-uncollapse probability $\tilde{P}_{\mathrm{S}}$ of observing both the result $m$ followed by a successful uncollapse is independent of $\rho_{\text {in }}$ - see Equation (32). Therefore, the combined effect of partial collapse and uncollapse brings no information about the initial state.

More quantitatively, we can use the same framework as at the end of Section 5.2 in order to track the information gain during the procedure. Suppose Plato assigns an initial distribution $\mathcal{P}_{k}$ of possible initial states as a statistical prior, to be updated as more information comes in. The measurement with result $m$ brings in this information, so Plato updates his prior to the posterior distribution $\mathcal{P}_{k}^{\prime}$ (see Equation (30)). Calculating in a similar way the distribution $\mathcal{P}_{k}^{\prime \prime}$ after the pair of the measurement and unmeasurement results, we find

$$
\mathcal{P}_{k}^{\prime \prime}=\frac{P_{\mathrm{S}}\left(\rho^{(k)}\right) \mathcal{P}_{k}^{\prime}}{\sum_{\tilde{k}} P_{\mathrm{S}}\left(\rho^{(\tilde{k})}\right) \mathcal{P}_{\tilde{k}}^{\prime}}=\frac{\tilde{P}_{\mathrm{S}}\left(\rho^{(k)}\right) \mathcal{P}_{k}}{\sum_{\tilde{k}} \tilde{P}_{\mathrm{S}}\left(\rho^{(k)}\right) \mathcal{P}_{\tilde{k}}},
$$

where $P_{\mathrm{S}}(\rho)$ and $\tilde{P}_{\mathrm{S}}(\rho)$ denote, respectively, the probabilities of uncollapsing (28) and a combined collapseuncollapse pair (32) for the initial state $\rho$. However, as we have already stressed, $\tilde{P}_{\mathrm{S}}(\rho)$ is independent of the initial state $\rho$, and therefore cancels out of the expression (35). This fact (and the normalisation of the prior $\left\{P_{k}\right\}$ ) restores the initial prior distribution, $\mathcal{P}_{k}^{\prime \prime}=\mathcal{P}_{k}$, and therefore Plato has learned nothing, thus avoiding the paradox. Reversing the logic, in order to avoid the paradox, $\tilde{P}_{\mathrm{S}}$ must be independent of the initial state, as found in Equations (32) and (33).

\subsection{Revisiting the previous uncollapse strategies}

With these general and abstract results in hand, we can now revisit the two examples discussed in Sections 3 and 4. Notice though that the principle drawback of the general results is that while they give the ideal success probability, they do not provide the strategy as to how achieve the upper bound.

The measurement situation in the case of the phase qubit (Section 3.1) may be described with a twooutcome POVM, with elements $E_{n}$ and $E_{y}$, where $n$ denotes the null result, and $y$ denotes the affirmative (tunnelling) result. The POVM elements, given in the $|0\rangle,|1\rangle$ basis are

$$
E_{n}=\left(\begin{array}{cc}
1 & 0 \\
0 & \exp (-\Gamma t)
\end{array}\right), E_{y}=\left(\begin{array}{cc}
0 & 0 \\
0 & 1-\exp (-\Gamma t)
\end{array}\right),
$$

with the obvious completeness relation $E_{n}+E_{y=1} 1$.

It is interesting to notice that while $E_{n}=M_{n}^{\dagger} M_{n}$ corresponds to a Kraus operator $M_{n}$ (see below), no meaningful Kraus operator $M_{y}$ can be introduced for 
the POVM element $E_{y}$, because in the case of a tunnelling event the system leaves its two-dimensional Hilbert space and becomes incoherent (so that a single Kraus operator cannot be introduced even in the extended Hilbert space). However, this is not important for us because we are interested in the nullresult case only. The null-result Kraus operator $M_{n}$ can be found by comparing the phase qubit dynamical Equations (1), (2) and (3) with the general state disturbance rule (24); this gives $M_{n}=\operatorname{diag}\{1$, $\exp (-\Gamma t / 2) \exp (-i \varphi)\}$.

It is easy to find that the uncollapsing strategy of Section 3.2 based on two null-result measurements is optimal in the sense that its success probability $P_{S}$ reaches the upper bound (28). The numerator of (28) is the smallest eigenvalue of $E_{n}$, which is $\exp (-\Gamma t)$, while the denominator is $\operatorname{Tr}\left(E_{n} \rho_{\text {in }}\right)=\rho_{\text {in, }, 00}+$ $\exp (-\Gamma t) \rho_{\text {in,11 }}$. Therefore, the bound (28) coincides with Equation (4), thus confirming the optimality of the analysed uncollapsing procedure.

The overall success probability $\tilde{P}_{\mathrm{S}}$, which is the joint probability of two null results is

$$
\tilde{P}_{\mathrm{S}}=\left(\rho_{\text {in }, 00}+\exp (-\Gamma) t \rho_{\text {in }, 11}\right) P_{\mathrm{S}}=\exp (-\Gamma t) .
$$

As expected (see Section 5.3) this probability does not depend on the initial state. It is easy to see that it also coincides with the upper bound (33) for $\tilde{P}_{\mathrm{S}}$.

While the analysed double-null-result uncollapsing strategy is optimal, an example of a non-optimal uncollapsing for a phase qubit was considered in [36]. It was shown that if the measurement process is performed simultaneously with Rabi oscillations, then in the null-result case the initial state is periodically restored. The non-optimality of uncollapsing for such a procedure is due to measurement of an evolving qubit, which corresponds to a sequence of many measurements; a similar reason for the non-optimality will be discussed at the end of Section 6 .

Now let us turn to the DQD charge qubit example of Section 4. First, the qubit measurement dynamics $[(5),(6)$ and (7)] can be related to the general POVMtype measurement formalism in the following way (similar to [32]). For a fixed time $t$ the measurement result $m$ can be associated with the averaged QPC current $\bar{I}$ (or, equivalently, with the dimensionless quantity $r$ ). The Kraus operator $M_{m}$ in the measurement basis $|1\rangle$ and $|2\rangle$ then should be chosen as $M_{m}=\operatorname{diag}\left\{\left[P_{1}(\bar{I})\right]^{1 / 2},\left[P_{2}(\bar{I})\right]^{1 / 2}\right\}$ in order to reproduce Equations (5), (6) and (7). Notice that $P_{m}$ in Equation (25) now describes the probability density of the result $\bar{I}$ instead of probability, because the measurement result becomes a continuous variable.

We can now check to see if the 'wait and stop' uncollapsing strategy of Section 4.2 is the optimal one by comparing the general upper bound (28) for the success probability $P_{\mathrm{S}}$ with the result (15). Substituting the probabilities in the bound (28) with probability densities, we find

$$
P_{\mathrm{S}} \leq \frac{\min \left\{P_{1}(\bar{I}), P_{2}(\bar{I})\right\}}{P_{1}(\bar{I}) \rho_{\mathrm{in}, 11}+P_{2}(\bar{I}) \rho_{\mathrm{in}, 22}},
$$

where $\bar{I}$ corresponds to the measurement result $r_{0}$. This bound coincides with Equation (15) because $\mathrm{P}_{1}(\bar{I}) /$ $\mathrm{P}_{2}(\bar{I})=\exp \left(2 \mathrm{r}_{0}\right)$, which proves the optimality of the discussed 'wait and stop' strategy. (Surely, there are numerous non-optimal uncollapsing strategies; for example, by stopping the measurement after the second crossing of the origin by $r(t)$.)

It is also instructive to not specify the result of the first measurement, but to find the total probability $\tilde{P}_{\mathrm{S}}^{\text {total }}$ (see Equation (34)) that the initial qubit state can be restored after a measurement for time $t$. This is given by averaging $P_{S}$ in Equation (15) over the results $r_{0}$ with the corresponding weights (11). This averaging is technically easier using the form of Equation (38) and gives

$$
\tilde{P}_{\mathrm{S}}^{\text {total }}=\int \mathrm{d} \bar{I} \min \left\{P_{1}(\bar{I}), P_{2}(\bar{I})\right\}=1-\operatorname{erf}\left(\left(\frac{t}{2 T_{M}}\right)^{1 / 2}\right),
$$

which depends only on the 'strength' $t / T_{M}=t(\Delta I)^{2} /$ $2 S_{I}$ of the first measurement, but not on the initial state, as expected from the discussion in Section 5.3. Notice that the result (39) reaches the upper bound (34) because (15) reaches the upper bound (28).

\section{Evolving charge qubit}

Armed with the general uncollapsing formalism of Section 5, we now turn to the case of the finite-Hamiltonian DQD measured by QPC, by including internal evolution of the qubit via a qubit Hamiltonian,

$$
H_{\mathrm{QB}}=-(\varepsilon / 2) \sigma_{z}+H \sigma_{x},
$$

where $\varepsilon$ is the energy asymmetry between the quantum dot levels, and $H$ is the tunnel coupling between the dots. In this case Equations (5)-(7) are no longer valid and should be replaced by the Bayesian equations [13] (in Stratonovich form [38])

$$
\begin{aligned}
\dot{\rho}_{11}=-\dot{\rho}_{22}= & -2 H \operatorname{Im} \rho_{12}+\rho_{11} \rho_{22} \frac{2 \Delta I}{S_{I}}\left[I(t)-I_{0}\right], \\
\dot{\rho}_{12}= & \mathrm{i} \varepsilon \rho_{12}+\mathrm{i} H\left(\rho_{11}-\rho_{22}\right)-\left(\rho_{11}-\rho_{22}\right) \\
& \times \frac{\Delta I}{S_{I}}\left[I(t)-I_{0}\right] \rho_{12},
\end{aligned}
$$


where $I(t)$ is the QPC current,

$$
I(t)=\rho_{11}(t) I_{1}+\rho_{22}(t) I_{2}+\xi(t),
$$

containing white noise $\xi(t)$ with spectral density $S_{I}$, and we use $\hbar=1$. These evolution equations are nonlinear and not very simple to deal with. To discuss the undoing of a continuous measurement, it is more convenient to use a non-normalised density matrix $\sigma$, which has an advantage of dealing with linear equations.

We rewrite Equations (41)-(42) in the form

$$
\begin{gathered}
\rho=\sigma / \operatorname{Tr} \sigma \\
\dot{\sigma}_{11}=-2 H \operatorname{Im} \sigma_{12}-\sigma_{11} \frac{1}{S_{I}}\left[I(t)-I_{1}\right]^{2}, \\
\dot{\sigma}_{22}=2 H \operatorname{Im} \sigma_{12}-\sigma_{22} \frac{1}{S_{I}}\left[I(t)-I_{2}\right]^{2}, \\
\dot{\sigma}_{12}=\mathrm{i} \varepsilon \sigma_{12}+\mathrm{i} H\left(\sigma_{11}-\sigma_{22}\right) \\
-\sigma_{12}\left\{\frac{\left[I(t)-I_{0}\right]^{2}}{S_{I}}+\frac{(\Delta I)^{2}}{4 S_{I}}\right\},
\end{gathered}
$$

so that $\sigma(0)=\rho(0)$, while the ratio $\sigma(t) / \rho(t)$ decreases with time and is equal to the normalised probability density of the corresponding realisation of the detector output $I\left(t^{\prime}\right), 0 \leq t^{\prime} \leq t .^{7}$ In the language of general quantum measurement this formulation corresponds to omitting the denominator in Equation (24). Notice that we still consider an ideal detector, so an initially pure state remains pure, $\left|\sigma_{12}\right|^{2}=\sigma_{11} \sigma_{22}{ }^{7}$

A casual inspection of Equations (45)-(47) shows that they are seemingly not well defined because the terms $\left[I(t)-I_{0,1,2}\right]^{2}$ contain the term $\xi(t)^{2}=\infty$ (from the relation $\left.\langle\xi(t) \xi(0)\rangle=\left(S_{I} / 2\right) \delta(t)\right)$. This divergence is artificial because there will always be a small correlation time $T$ of the noise and/or a finite detector bandwidth $B$ (corresponding to $T=1 / 4 B$ ), so there will be a large but finite constant $\mathcal{C}=\left\langle\xi(t)^{2}\right\rangle=S_{I} / 4 T$ contained in terms of the form $\left[I(t)-I_{0,1,2}\right]^{2}$. It is easy to see that Equations (45)-(47) do not change if we subtract the same constant from these terms $\left[I(t)-I_{0,1,2}\right]^{2} \rightarrow\left[I(t)-I_{0,1,2}\right]^{2}-\mathcal{C}$. This can be shown by considering another unnormalised density matrix $\eta=\sigma \exp (t / T)$. Writing the linear Bayesian equations (45)-(47) in the form $\dot{\sigma}_{i j}=f_{i j}[\sigma]$, the equations transform to $\dot{\eta}_{i j}=f_{i j}[\eta \exp (-t / T)] \exp (t / T)+\eta_{i j} / T$ under the change of variables. The unnormalised Bayesian equations are linear in the density matrix elements $\sigma_{i j}$, so the exponential factors cancel out. The new equations are thus the same as the old ones with a constant $\mathcal{C}=S_{I} / 4 T$ subtracted from the $\left[I(t)-I_{0,1,2}\right]^{2}$ terms. The unspecified constant $T$ in the density matrix transformation may be chosen to be the short correlation time $T$ discussed above, thus cancelling the large term and making Equations (45)-(47) well defined. The only price to be paid for this transformation is an altered normalisation, that will cancel in the normalised density matrix (44).

For a particular realisation of the detector output $I\left(t^{\prime}\right), 0 \leq t^{\prime} \leq t$, Equations (45)-(47) define a linear map $\sigma(0) \rightarrow \sigma(t)$, corresponding to a particular Kraus operator $M_{m}$ (which, therefore, can be denoted as $\left.M_{\left\{I_{\}}\right.}\right)$. For the uncollapsing we have to realise the map, corresponding to the inverse Kraus operator $\mathrm{CM}_{\{I\}}^{-1}$ (see Section 5.1). It is obvious that in contrast to the case of the non-evolving qubit discussed in Section 4, this cannot be done by simply continuing the measurement and waiting for a specific result. The reason is that now the map is characterised by six real parameters (eight parameters for a linear operator $\mathrm{CM}_{\{1\}}^{-1}$ with neglected overall phase and normalisation), instead of one parameter for the non-evolving case (see Equations (7) and (9)). We will discuss a little later how the six-parameter uncollapsing procedure can be realised explicitly. Before that we discuss how to find the operator $M_{\left\{I_{\}}\right.}$in a more straightforward way, from Equations (45)-(47).

Let us consider only the evolution of (unnormalised) pure states $|\psi(t)\rangle=\alpha(t)|1\rangle+\beta(t)|2\rangle$, so that $\sigma=|\psi\rangle\langle\psi|$. Then Equations (45)-(47) can be rewritten as

$$
\begin{aligned}
& \dot{\alpha}=+\mathrm{i} \frac{\varepsilon}{2} \alpha-\mathrm{i} H \beta-\alpha \frac{1}{2 S_{I}}\left[I(t)-I_{1}\right]^{2}, \\
& \dot{\beta}=-\mathrm{i} \frac{\varepsilon}{2} \beta-\mathrm{i} H \alpha-\beta \frac{1}{2 S_{I}}\left[I(t)-I_{2}\right]^{2},
\end{aligned}
$$

where the infinite part of $I^{2}(t)$ can be cancelled in the same way as discussed above. The linearity of these equations guarantees that for any given realisation of $I(t)$, it is sufficient to solve (48) and (49) for the initial states $|1\rangle$ and $|2\rangle$ in order to find the solution for an arbitrary initial state of the qubit. Defining $\boldsymbol{v}_{1}=$ $\alpha_{1}(t)|1\rangle+\beta_{1}(t)|2\rangle$ as the solution of (48) and (49) for initial state $|1\rangle$, and $\boldsymbol{v}_{2}=\alpha_{2}(t)|1\rangle+\beta_{2}(t)|2\rangle$ as the solution of (48) and (49) for initial state $|2\rangle$, we can write the solution for an arbitrary initial state $\left|\psi_{\text {in }}\right\rangle=$ $|\psi(0)\rangle=a|1\rangle+b|2\rangle$ as $|\psi(t)\rangle=a v_{1}+b v_{2}$. Therefore, the Kraus operator $M_{\{l\}}$ for a given realisation of $I(t)$, in the $|1\rangle,|2\rangle$ basis is

$$
M_{\{I\}}=\left(\begin{array}{ll}
\alpha_{1}(t) & \alpha_{2}(t) \\
\beta_{1}(t) & \beta_{2}(t)
\end{array}\right) .
$$

For the uncollapsing we need to apply the Kraus operator $\mathrm{CM}_{\{I\}}^{-1}$, which maps the state $\boldsymbol{v}_{1}$ onto $C|1\rangle$ and the state $\boldsymbol{v}_{2}$ onto $C|2\rangle$. The reason why we need a nonunitary transformation is that the vectors $\boldsymbol{v}_{1}$ and $\boldsymbol{v}_{2}$ are 
in general non-orthogonal and have different norms. Geometrically, such a transformation can be done by using a relative shrinking or stretching of two orthogonal axes (found for a given $M_{\left\{I_{\}}\right.}$), which would make $\boldsymbol{v}_{1}$ and $\boldsymbol{v}_{2}$ orthogonal and equal in norm, followed by a unitary transformation (this would correspond to the decomposition of the form $U E^{1 / 2}$ see Section 5.1). However, for a practical realisation of uncollapsing it is most natural to use the shrinking or stretching of the axes $|1\rangle$ and $|2\rangle$ via a continuous QND measurement with the QPC in the way considered above for a non-evolving qubit. In this case the uncollapsing procedure can be done in three steps (see Section 5.1): unitary evolution $V$, continuous QND measurement (where the qubit Hamiltonian is turned off, $\varepsilon=H=0$ ) described by a diagonal matrix $L$, and a final unitary operation $U$ (in the notation of Section 5.1, $V$ corresponds to $V_{L}^{\dagger} U_{m}^{\dagger}$, and $U$ corresponds to $U_{L}^{\dagger}$ ). These operators should satisfy

$$
U L V=C M_{\{I\}}^{-1},
$$

and it is easy to find $U, L$, and $V$ explicitly by recognising Equation (51) as a singular value decomposition of the operator $C M_{\{I\}}^{-1}$ (recall here that $L$ is diagonal; also notice that the standard form for the singular value decomposition is slightly different, with $V$ denoted as $V^{\dagger}$ ).

To find $L$ explicitly, we notice that

$$
C^{-2} M_{\{I\}}^{\dagger} M_{\{I\}}=U L^{-2} U^{\dagger},
$$

which is simply the diagonalisation of $C^{-2} M_{\{I\}}^{\dagger} M_{\{I\}}$. Therefore,

$$
L=C\left(\begin{array}{cc}
\lambda_{-}^{-1 / 2} & 0 \\
0 & \lambda_{+}^{-1 / 2}
\end{array}\right) \text { or } L=C\left(\begin{array}{cc}
\lambda_{+}^{-1 / 2} & 0 \\
0 & \lambda_{-}^{-1 / 2}
\end{array}\right)
$$

where

$$
\begin{aligned}
\lambda_{ \pm}= & \frac{\left\|v_{1}\right\|^{2}+\left\|v_{2}\right\|^{2}}{2} \\
& \pm\left[\left(\frac{\left\|v_{1}\right\|^{2}-\left\|v_{2}\right\|^{2}}{2}\right)^{2}+\left|\boldsymbol{v}_{1} \cdot v_{2}^{*}\right|^{2}\right]^{1 / 2}
\end{aligned}
$$

are the eigenvalues of the operator $M_{\{I\}}^{\dagger} M_{\{I\}}$ and the vectors $\boldsymbol{v}_{\mathrm{i}}$ are defined above Equation (50). The Cauchy-Schwartz inequality, $\left|\boldsymbol{v}_{1} \cdot \boldsymbol{v}_{2}^{*}\right|^{2} \leq\left\|\boldsymbol{v}_{1}\right\|^{2}\left\|\boldsymbol{v}_{2}\right\|^{2}$, guarantees the non-negativity of $\lambda_{-}$. (The notation $\boldsymbol{v}_{1} \cdot \boldsymbol{v}_{2}$ is used for the inner product $\left\langle v_{2} \mid v_{1}\right\rangle$ of $\boldsymbol{v}_{2}$ and $\boldsymbol{v}_{1}$.)

To find $U$, we use Equation (52) again and see that the columns of $U$ are composed of the eigenvectors of $\mathrm{M}_{\{I\}}^{\dagger} \mathbf{M}_{\{I\}}$ (the sequence of columns depends on the choice in Equation (53)). Finally, $V$ is given by
$V=U^{\dagger} C L^{-1} \mathrm{M}_{\{I\}}^{-1}$. For brevity we will not show the matrices $U$ and $V$ explicitly.

In the physical realisation of the uncollapsing procedure the measurement step $L$ can be performed in exactly the same way as in Section 4.2. Comparing Equation (53) with Equations (9) and (7), we see that the continuous measurement by the QPC should be stopped when the dimensionless measurement result $r(t)$ reaches the value

$$
r_{1}=\ln \left(\lambda_{+} / \lambda_{-}\right)^{1 / 2}>0 \text { or } r_{2}=\ln \left(\lambda_{-} / \lambda_{+}\right)^{1 / 2}<0,
$$

for the first and second choice in Equation (53), respectively (the choice should be made beforehand, since it determines operation $V$ ). As previously mentioned, the constant $C$ is not important here because the physical state is always normalised. The procedure fails if the desired result is not reached during the continuous measurement.

The unitary operations $V$ and $U$ can be practically realised in three substeps each: $z$-rotation on the Bloch sphere by applying non-zero energy asymmetry $\varepsilon$ for some time, $y$-rotation by applying non-zero tunnelling $H$, and then one more $z$-rotation. However, the last $z$-rotation of $V$ and the first $z$-rotation of $U$ are simply added to each other (since $L$ does not change the relative phase of the state components or, equivalently, the azimuth angle on the Bloch sphere). The corresponding trivial degree of freedom can be eliminated, for example, by realising the operation $V$ in only two substeps, without the second $z$-rotation.

Let us count the number of real parameters, characterising the uncollapsing procedure. Since $V$ and $U$ together provide $2 \times 3-1=5$ parameters, and the desired result $r$ in the measurement step adds one more parameter, the overall number of parameters is 6 . As expected, this is exactly the needed number of parameters characterising an arbitrary Kraus operator for the qubit (neglecting normalisation and overall phase). Let us also mention the fact from linear algebra that the singular value decomposition (51) is unique in the non-degenerate case $\left(\lambda_{+}>\lambda_{-}>0\right)$, up to the permutation of singular values (corresponding to the choice in Equation (53)) and arbitrary phase factors in columns of $U$, with compensating changes in $V$ (this corresponds to the previously discussed compensation of the $z$-rotations).

Now let us discuss the probability $P_{\mathrm{S}}$ of the successful uncollapsing. From the general theory of Section 5 (Equation (23)), it is bounded from above by a fraction, $P_{\mathrm{S}} \leq \min P_{\left\{I_{\}}\right.} / P_{\left\{I_{\}}\right.}\left(\left|\psi_{\text {in }}\right\rangle\right)$, in which the denominator is the probability density of the given realisation $I(t)$ for the initial state $\left|\psi_{\text {in }}\right\rangle=a|1\rangle+b|2\rangle$ (with $|a|^{2}+|b|^{2}=1$ ), while the numerator is this probability minimised over all initial states. So, the 
denominator is given by the squared norm of the final state $|\psi(t)\rangle=a \boldsymbol{v}_{1}+b \boldsymbol{v}_{2}$

$$
P_{\{I\}}\left(\left|\psi_{\text {in }}\right\rangle\right)=\left\|a v_{1}+b \boldsymbol{v}_{2}\right\|^{2},
$$

while the numerator is given by minimising (56) over all normalised initial states. It is easy to see that this minimum is equal to the minimum eigenvalue $\lambda_{-}$ of the operator $\mathbf{M}_{\{I\}}^{\dagger} \mathrm{M}_{\{I\}}$, given by Equation (54); therefore,

$$
P_{\mathrm{S}} \leq \frac{\lambda_{-}}{\left\|a \boldsymbol{v}_{1}+b \boldsymbol{v}_{2}\right\|^{2}} .
$$

Converting this result into the language of density matrices and simultaneously generalising it to an arbitrary initial state $\rho_{\text {in }}$, we obtain the bound

$$
P_{\mathrm{S}} \leq \frac{\frac{\left\|\boldsymbol{v}_{1}\right\|^{2}+\left\|\boldsymbol{v}_{2}\right\|^{2}}{2}-\left[\left(\frac{\left\|\boldsymbol{v}_{1}\right\|^{2}-\left\|\boldsymbol{v}_{2}\right\|^{2}}{2}\right)^{2}+\left|\boldsymbol{v}_{1} \cdot \boldsymbol{v}_{2}^{*}\right|^{2}\right]^{1 / 2}}{\rho_{\mathrm{in}, 11}\left\|\boldsymbol{v}_{1}\right\|^{2}+\rho_{\mathrm{in}, 22}\left\|\boldsymbol{v}_{2}\right\|^{2}+2 \operatorname{Re}\left[\rho_{\mathrm{in}, 12} \boldsymbol{v}_{1} \cdot \boldsymbol{v}_{2}^{*}\right]},
$$

in which the numerator is the explicit expression (54) for $\lambda_{-}$. It is easy to check that this result reduces to the bound (38) in the non-evolving case, in which $\boldsymbol{v}_{1}=\left(\left[\mathrm{P}_{1}(\bar{I})\right]^{1 / 2}, 0\right)^{\mathrm{T}}$ and $\boldsymbol{v}_{2}=\left(0,\left[P_{2}(\bar{I})^{\mathrm{I} / 2}\right)^{\mathrm{T}}\right.$.

The uncollapsing procedure discussed in this section is optimal in the sense that it corresponds to the upper bound of Equation (58). To prove this statement, instead of calculating $P_{\mathrm{S}}$ explicitly, let us use the fact (see Section 5.3) that the product $P_{\mathrm{S}} P_{\left\{I_{\}}\right.}$ cannot depend on the initial state. Therefore, it is sufficient to prove the optimality of $P_{\mathrm{S}}$ only for one initial state. Let us choose the state $\left|\psi_{\text {in }}\right\rangle$ that is the eigenvector of $\mathrm{M}_{\{I\}}^{\dagger} \mathbf{M}_{\{I\}}$, corresponding to the eigenvalue $\lambda_{-}$. Then after the first measurement (operator $\left.M_{\{I\}}\right)$ and the unitary operation $V$ it is transformed into one of the basis states $(|1\rangle$ or $|2\rangle$ for the first or second choice in (53), respectively). Recall that for the non-evolving (QND) measurement case, $r(t) \rightarrow \infty$ for the initial state $|1\rangle$, while $r(t) \rightarrow-\infty$ for the initial state $|2\rangle$. The crossing thresholds (55) indicate that the measurement $L$ is always successful because $r(t)$ necessarily crosses the desired value (which is positive for $|1\rangle$ and negative for $|2\rangle$, as discussed above). Therefore, the uncollapsing success probability for this special state is $100 \%$, that is equal to the upper bound (58). As mentioned above, the optimality of the procedure for this special state also proves the optimality for any initial state.

Obviously, an uncollapsing procedure can also be non-optimal. As an example, let us consider a procedure which realises the desired mapping $\left\{\boldsymbol{v}_{1}, \boldsymbol{v}_{2}\right\} \rightarrow$ $\{C|1\rangle, C|2\rangle\}$ using two measurements instead of one. The goal of the first measurement is to map $\left\{\boldsymbol{v}_{1}, \boldsymbol{v}_{2}\right\}$ into an orthogonal pair of vectors, while the goal of the second measurement is to equalise their norms, keeping them orthogonal. The first goal can be achieved by stretching/shrinking of the Hilbert space along any axis $u$ of the form $\boldsymbol{v}_{1}+c \boldsymbol{v}_{2}\left(\boldsymbol{v}_{1} \cdot \boldsymbol{v}_{2}\right) /\left|\boldsymbol{v}_{1} \cdot \boldsymbol{v}_{2}\right|$ with an arbitrary positive real number $c$ (it is easy to visualise this procedure of making two vectors orthogonal by assuming the space of real vectors, for which the axis $u$ is geometrically in between $\boldsymbol{v}_{1}$ and $\boldsymbol{v}_{2}$; the same geometrical idea works for complex vectors). We recall that measurement for a non-evolving qubit (Section 4.1) stretches (squeezes) the $|1\rangle$ axis, while squeezing (stretching) the $|2\rangle$ axis. Therefore, the first goal can be achieved by a unitary operation which rotates $u$ into $|1\rangle$, followed by a continuous measurement (with a QPC) of a non-evolving qubit, to be stopped when the mapped vectors become orthogonal. After the vectors $\left\{\boldsymbol{v}_{1}, \boldsymbol{v}_{2}\right\}$ are transformed into an orthogonal pair by the (successful) first measurement, the second part of the procedure should stretch/shrink the 2D Hilbert space along the resulting vectors to make them equal in norm. This can be done similarly, by a unitary rotation and partial measurement of a non-evolving qubit. Finally, another unitary operation can be used to map the resulting pair of vectors into $\{C|1\rangle, C|2\rangle\}$, thus completing the uncollapsing procedure. Notice that both measurements are performed in the 'wait and stop' manner, and both measurements should be successful to realise the uncollapsing. While the successfully uncollapsed state is still perfect in this procedure, the probability of success is lower than the bound (58). To prove this non-optimality, let us again use the initial eigenstate $\left|\psi_{\text {in }}\right\rangle$, which corresponds to eigenvalue $\lambda_{-}$, so that the bound (58) is $100 \%$. Then the probability of success for the first measurement is in general less than $100 \%$ (it is $100 \%$ only for one specific axis discussed previously, while here we consider a range of possible axes by allowing $c$ to vary). Thus, the success probability is less than $100 \%$ for this special state, and therefore $P_{\mathrm{S}}$ is below the bound (58) for any initial state.

\section{General procedure for entangled charge qubits}

Let us present an explicit procedure [14] which can be used in principle to undo an arbitrary measurement $M_{m}$ of any number $N$ of entangled qubits with maximum probability of success $P_{\mathrm{S}}$. For simplicity we consider double-quantum-dot charge qubits and assume that any unitary transformation can be used in the procedure. If the operator $M_{m}$ was produced by a one-qubit measurement, and other entangled qubits were not experiencing a Hamiltonian evolution, then the formalism of Section 4.1 is essentially unchanged [40], and uncollapsing of the measured qubit leads to 
the restoration of the whole entangled state. If the operator $M_{m}$ was produced by a one-qubit measurement, while other qubits were evolving in a unitary way but not interacting with the measured qubit, then uncollapsing is also easy: we should uncollapse the measured qubit in the usual way (Section 4.2) and should apply the inverse unitary transformation for the other qubits. In this section, however, we do not consider these simple special cases; the goal is to undo an arbitrary Kraus operator $M_{m}$.

Let us decompose $M_{m}$ as $M_{m}=U_{m} E_{m}^{1 / 2}$ (see Equation (18)). Reversing the unitary operation $U_{m}$ can be done in the regular Hamiltonian way, so the nontrivial part is undoing the $E_{m}^{1 / 2}$ operator. We recall the diagonalisation of $E_{m}$ is given by $E_{m}=\sum_{i} p_{i}^{(m)}|i\rangle\langle i|$ with vectors $|i\rangle$ forming an orthonormal basis. As discussed in Section 5.1, for the optimal uncollapsing which maximises the success probability, we have to perform a procedure corresponding to the measurement operator $\tilde{L}=\left(\min _{j} p_{j}^{(m)}\right)^{1 / 2} E_{m}^{-1 / 2}$ which is also diagonal in the basis $|i\rangle$ with corresponding matrix elements $\tilde{L}_{i i}=\langle i|\tilde{L}| i\rangle=\left[\left(\min _{j} p_{j}^{(m)}\right) / p_{i}^{(m)}\right]^{1 / 2}$, all of which are between 0 and 1 . Given $N$ qubits, $i$ ranges from 1 to $2^{N}$. Notice that $L$ is obviously Hermitian.

Our procedure is to realise $\tilde{L}$ with a sequence of null-result measurements and unitary operations. Shown in Figure 3 is an illustration of the physical set-up that is used for the measurements: a QPC (tunnel junction) capacitively coupled to $N$ nonevolving DQD charge qubits. We assume that the QPC is tuned to a highly nonlinear regime, for which no electron can tunnel across the QPC barrier on experimentally relevant time-scales unless all qubits are in the state $|1\rangle$. We name this multi-qubit state $|1\rangle \equiv|1,1, \ldots, 1\rangle$. Such a regime is possible because of the exponential dependence of the tunnelling rate on QPC barrier height, while the barrier height depends linearly on the states of the coupled qubits. Of course, this regime is not quite realistic; however, we discuss the procedure in principle. We also assume that

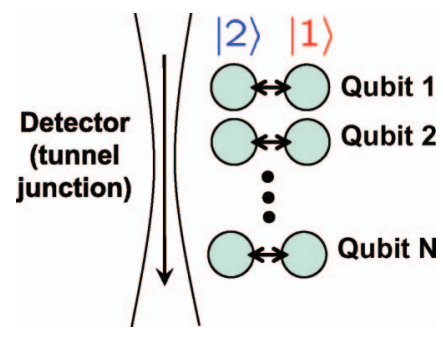

Figure 3. Schematic set-up for uncollapsing of $N$ entangled qubits. The tunnel junction detector (QPC) is in a strongly nonlinear regime, so that an electron can tunnel through it with rate $\gamma$ only when all qubits are in state $|1\rangle$. even for the $N$-qubit state $|1\rangle$, the rate $\gamma$ of electron tunnelling through the QPC is rather low, so that we can distinguish single tunnelling events (technically, this would require an additional single-electron transistor). If we perform the measurement during time $t$ and see no tunnelling through the QPC, then similarly to the case of Section 3.1, the corresponding null-result Kraus operator $M_{n}$ shrinks the $|1\rangle$ axis of the Hilbert space by the factor $\exp (-\gamma t / 2)$, while leaving all perpendicular axes unchanged. For the matrix elements this means $\left\langle\mathbb{1}\left|M_{n}\right| \mathbb{1}\right\rangle=\exp (-\gamma t / 2)$, $\left\langle\psi_{j}^{\perp}\left|M_{n}\right| \psi_{j^{\prime}}^{\perp}\right\rangle=\delta j j^{\prime},\left\langle\psi_{j}^{\perp}\left|M_{n}\right| 1\right\rangle=\left\langle 1 \mid M_{n}+\psi_{j}^{\perp}\right\rangle=0$, where we introduced a set of $2^{N}-1$ states $\left|\psi_{j}^{\perp}\right\rangle$ spanning the subspace orthogonal to $|1\rangle$.

The general strategy to implement the operator $\tilde{L}$ is the following. We first note that in the basis $|i\rangle$ that diagonalises $\tilde{L}$, this diagonal matrix can be represented as a product of $2^{N}$ diagonal matrices, where each term in the product has all diagonal entries as 1, except the $i$ th entry: $\operatorname{diag}\left\{1,1, \ldots, \tilde{L}_{i i}, \ldots, 1\right\}$. Each of these matrices may be interpreted as a separate Kraus operator that can be sequentially implemented. Thus, the explicit physical procedure consists of $2^{N}$ steps, each of which has three substeps. First, we apply a unitary transformation $U_{1}$ which transforms the first basis vector $|i=1\rangle$ into the state $|1\rangle$. Then the evolution of all qubits is stopped, and the detector is turned on for a time $t_{1}$. This time is chosen so that the null-result Kraus operator $L^{(1)}$ has the desired matrix element $\langle 1|L(1)| 1\rangle=L_{11}$; this condition yields $\mathrm{t}_{1}=-2 \gamma^{-1} \ln \tilde{L}_{i i}$. The measurement is then followed by the reverse unitary, $U_{i}^{\dagger}$, to take the state $|1\rangle$ back to state $|i=1\rangle$. This three-substep procedure is then repeated for $i=2,3, \ldots, 2^{N}$, sequentially transforming the state $|i\rangle$ to $|1\rangle$ with unitary $U_{i}$, and performing measurement with the detector for a time $t_{i}=-2 \gamma^{-1} \ln$ $\tilde{L}_{i i}$, followed by the reverse unitary, $U_{i}^{\dagger}$. This sequence of steps decomposes the uncollapsing operator $\tilde{L}$ as

$$
\tilde{L}=U_{2^{N}}^{\dagger} L^{\left(2^{N}\right)} U_{2^{N}} \ldots U_{2}^{\dagger} L^{(2)} U_{2} U_{1}^{\dagger} L^{(1)} U_{1} .
$$

The uncollapsing procedure is successful only if there were no tunnelling events in the QPC. By construction, the success probability $P_{\mathrm{S}}$ for this procedure maximises the general bound (28).

The success probability $P_{\mathrm{S}}$ for the uncollapsing process $\rho_{m} \rightarrow \rho_{\text {in }}$ with $\rho_{\text {in }}=\tilde{L} \tilde{\rho} \tilde{L}^{\dagger} / \operatorname{Tr}\left(\tilde{L}^{\dagger} \tilde{L} \tilde{\rho}\right)$ and $\tilde{\rho}=U_{m}^{\dagger} \rho_{m} U_{m}$, can be calculated as

$$
P_{\mathrm{S}}=\operatorname{Tr}\left(\tilde{L}^{\dagger} \tilde{L} \tilde{\rho}\right)=\sum_{i} \tilde{L}_{i i}^{2} \tilde{\rho} i i=\sum_{i} \tilde{\rho}_{i i} \exp \left(-\gamma t_{i}\right),
$$

where $\tilde{\rho}_{i j}$ are the matrix elements of $\tilde{\rho}$ in the basis $|i\rangle$, which diagonalises $\tilde{L}$. We may also find this result from another perspective by realising that the success 
probability is simply the product of the null-result probabilities $p_{\mathrm{S}}^{(i)}$ of all $2^{N}$ measurements,

$$
P_{\mathrm{S}}=\prod_{i}^{2^{N}} P_{\mathrm{S}}^{(i)}, p_{\mathrm{S}}^{(i)}=\frac{\sum_{j=1}^{i} \tilde{\rho}_{j j} \exp \left(-\gamma t_{j}\right)+\sum_{j=i+1}^{2^{N}} \tilde{\rho}_{j j}}{\sum_{j=1}^{i-1} \tilde{\rho}_{j j} \exp \left(-\gamma t_{j}\right)+\sum_{j=i+1}^{2^{N}} \tilde{\rho}_{j j}},
$$

where the expression for $p_{\mathrm{S}}^{(i)}$ comes from comparing the traces of unnormalised density matrices after each of $2^{N}$ steps of the procedure. It is instructive to show explicitly that this expression for $\mathrm{p}_{\mathrm{S}}^{(i)}$ is equal to the expected expression

$$
p_{\mathrm{S}}^{(i)}=1-\left[1-\exp \left(-\gamma t_{i}\right)\right] \tilde{\rho}_{i i}^{(i)},
$$

in which $\tilde{\rho}^{(i)}$ is the normalised density matrix before the $i$ th step of the procedure (after $i-1$ null-result steps). This can be done if we prove the relation

$$
\prod_{i=1}^{k}\left[1-\tilde{\rho}_{i i}^{(i)}\left(1-\exp \left(-\gamma t_{i}\right)\right)\right]=1-\sum_{i=1}^{k}\left[1-\exp \left(-\gamma t_{i}\right)\right] \tilde{\rho}_{i i},
$$

(notice that the right-hand side of this equation is equal to the numerator in Equation (61) with substitution $k \rightarrow i$ ). Equation (63) can be proven by induction using the relation $\tilde{\rho}_{i i}^{(i)}=\tilde{\rho}_{i i} / \prod_{j=1}^{i-1}[1-$ $\left.\left[1-\exp \left(-\gamma t_{j}\right)\right] \tilde{\rho}_{j j}^{(j)}\right]$, which can be easily derived recursively, $\tilde{\rho}_{i i}^{(j)} \rightarrow \tilde{\rho}_{i i}^{(j+1)}$, starting from $\tilde{\rho}_{i i}^{(1)}=\tilde{\rho}_{i i}$. In this way we show consistency between the null-result probabilities given by Equations (61) and (62), permitting the calculation of $P_{\mathrm{S}}$ in two independent ways.

Let us mention again that the uncollapsing procedure considered in this section reaches the upper bound (28) for the success probability $P_{\mathrm{S}}$, that can be seen both by construction and explicitly.

\section{Recent developments in wavefunction uncollapse}

Before concluding, we wish to give a summary of some interesting recent developments in this area of research. We will briefly discuss two theory proposals and one experiment.

\subsection{Spin qubit}

The examples given above mainly concern quantum dot charge qubits. It is a natural question if a similar kind of partial collapse/uncollapse can be carried over to spin qubits. An analysis of this situation was carried out by Trauzettel, Burkard, and one of the authors [41]. There it was shown how an uncollapse measurement can be realised using a scheme similar to the recent experiments by Koppens et al. [42]. The essential idea of the spin-qubit experiments [42-45] is to manipulate and measure the spin of a single electron through the charge degree of freedom. This technique circumvents the otherwise difficult problem of controlling the weakly interacting spin. While we refer the reader to [41-45] for the details, we will give a simplified thumb-nail sketch of the physics here.

The qubit is encoded with two electron spins, where each electron is confined in a separate quantum dot. In contrast to our charge qubit discussion, these dots are open, with electrons able to enter and leave. Electrical bias is applied across this double quantum dot leading to charge transport. Electrons can tunnel sequentially, but spin blockade [46] restricts transport to situations where the two electrons form a spin singlet $(0,2) S$ on the right dot while the spin triplet $(0,2) T$ is outside the transport energy window due to the large single quantum dot exchange energy (here $(n, m)$ refers to $n$ electrons on the left dot and $m$ electrons on the right dot). This blockade physics provides an interesting initialisation procedure of the quantum register - when the single-electron current stops flowing, we are confident that the two-electron state is in a $(1,1) T$ state, because in the absence of spin flip processes, the tunnelling transition to the $(0$, 2) state is forbidden. From this configuration, it is possible to manipulate the system by applying electron spin resonance pulses [42], transitioning the state to have overlap with the singlet state. Thus, the electron on the left dot may tunnel (with rate $\Gamma$ ) to the right dot and exit the system, giving rise to a small electrical current at the drain when this process is repeated many times. Of course, this will happen with some probability controlled by the overlap of the state with the singlet.

Drawing on our experience with the phase qubit (see Section 3), it is clear how to devise a weak measurement experiment and an uncollapsing experiment: the allowed transition can be permitted for a time of one's choosing and then forbidden by detuning the energy levels with a voltage pulse to one of the quantum dot's gates. In this way one can weakly probe the two-electron state, and in the nullresult case (no single electron tunnelling) partially collapse it to the triplet subspace. In order to propose the uncollapse part of the experiment, it is easiest to consider the case when the surrounding nuclear spins [42] quickly admix the singlet state with a triplet state, permitting the two-qubit state to encode one effective qubit: parallel or anti-parallel spins. The weak measurement technique described above will then partially collapse the state toward the parallel state under a null-measurement (no single electron tunnelling). If now a $\pi$-pulse is applied to one of the spins with electron spin resonance, followed by a second null-measurement, this was shown to uncollapse the state of the effective qubit [41]. 


\subsection{Optical polarisation qubit}

Another interesting development is the experimental implementation of wavefunction uncollapse for optical qubits using the polarisation degree of freedom of single photons by Kim et al. [22] The weak measurement was implemented by passing the photon through a glass plate oriented at the Brewster angle. Only the vertical polarisation is reflected off of the glass plate (with some probability). By placing a single-photon detector where the photon would have gone had it reflected, a null-click measurement partially collapses the polarisation state to the horizontal polarisation. The strength of the measurement can be increased by placing a series of plates in a row, effectively increasing the net probability of a vertically-polarised photon reflecting at some point.

The wavefunction uncollapse is done by inserting a half-wave plate (exchanging the amplitude of horizontal with vertical polarisation), and having the same number of plates traversed by the photon again. If none of the single-photon detectors click, the polarisation state is uncollapsed. This has been verified [22] with quantum state tomography (with polariser and single-photon counter placed after all of the reflecting plates) on the photon, conditioned on none of the other photon detectors firing. The experiment showed an uncollapsing fidelity of above $94 \%$ for measurement strengths up to 0.9 . It was also pointed out that the information from the first weak measurement can be used for developing guessing strategies about the unknown initial state. Two such strategies were presented, and one was shown to be optimal. Of course, in the case where the measurement was subsequently undone, these strategies did no better than random guessing.

\subsection{Decoherence suppression by uncollapsing}

It has been recently shown [23] that uncollapsing can be used to suppress qubit decoherence due to energy relaxation at low temperature (this is a nearly dominant decoherence process in superconducting phase qubits [24] and the dominant process in superconducting 'transmon' qubits [47]). The proposed procedure is very close to the existing experiment [21] with the phase qubit. To protect the qubit state from energy relaxation, it is first partially measured with the strength $p_{t}=1-\exp (-\Gamma t)$ (see Section 3.1), so that in the null-result case the qubit state moves towards the ground state $|0\rangle$. The qubit is kept there during the storage/protection period, and then it is uncollapsed in the usual way (using a $\pi$-pulse, another null-result partial measurement and one more $\pi$-pulse - see Section 3.2), though the strength $\tilde{p} t$ of the second measurement can differ from $p_{t}$. The calculations show [23] that this procedure can significantly increase the quantum memory fidelity, and theoretically it can be made arbitrarily close to $100 \%$ even for a significant energy relaxation during the storage period. This is because the uncollapsing procedure preferentially selects the cases without energy relaxation. For the initial state $|0\rangle$ the energy relaxation is absent by itself, while for the initial state $|1\rangle$ the operating principle is the following: the first measurement keeps it as $|1\rangle$, but if the state jumps down to $|0\rangle$ during the storage period, then most likely there will be tunnelling during the second measurement, and therefore such events will be eliminated by the selection of only null-result cases. This is surely a simplified explanation, but the same idea works in the rigorous calculations as well. Notice though that stronger decoherence suppression requires a stronger measurement and therefore a smaller probability of selection. Estimates show that a significant decoherence suppression by uncollapsing can be demonstrated with present-day phase qubits.

\section{Conclusion}

We have reviewed and extended recent developments in the theory (and experiment) of wavefunction uncollapse by undoing quantum measurements. We have formulated the problem of wavefunction uncollapse in terms of a contest between the uncollapse proponent, Plato, and the uncollapse skeptic, Socrates, monitored by the arbiter, Aristotle. Plato claims to have the ability to uncollapse wavefunctions, and this ability can be tested under the rules of the contest set forth.

We have discussed several general features of the uncollapse process in the abstract case, such as the upper bound on the success probability and quantum information aspects of the problem. In order to probabilistically undo the measurement, it is necessary to erase the information extracted about the state in the first measurement. This is a necessary condition to uncollapse the wavefunction, because otherwise various paradoxes arise. However, the information erasure is surely not a sufficient condition: the unitary evolution should also be properly reversed and, as the most experimentally challenging condition, the process should not bring decoherence, which requires a very good (ideal) detector.

In addition to discussing the theory of wavefunction uncollapse in the abstract case, we have also considered a variety of solid-state implementations and specific practical strategies for wavefunction uncollapse. The cases of the phase qubit, the charge qubit (with and without Hamiltonian dynamics), and many entangled charge qubits have been examined 
in detail. Additionally, we have also discussed two experimental realisations of this physics, based on the phase qubit and the polarisation qubit, both of which have clearly demonstrated wavefunction uncollapse with high fidelity.

The ideality of the detector is necessary for perfect uncollapsing, and we have only dealt with these kinds of detectors in the theory section of this paper (by this we mean the detector adds no extra decoherence to the system). If a detector is slightly non-ideal, then even a perfectly executed uncollapse strategy will result in a slight infidelity in the final state. This is indeed the case in the experiments mentioned above although the fidelity was quite high. In such a situation there are two characteristics to contend with: the fidelity of uncollapsing as well as the probability of claimed success. It is an open topic for future research how these characteristics are related.

\section{Acknowledgements}

The work was supported by NSA and IARPA under ARO Grant W911NF-08-1-0336, the National Science Foundation under Grant No. DMR-0844899, and the University of Rochester.

\section{Notes}

1. One of the possible procedures is the following. If the post-measured state is not pure, we can apply more measurements to make it pure, and then probabilistically apply a unitary operation from an easily calculable set, which creates a mixture of pure states identical to the initial state.

2. An exception is when the initial state belongs to a very limited set, in which the measurement result corresponds to only one state.

3. The classical Bayes rule $\mathcal{P}(k \mid m)=\mathcal{P}(m \mid k) \mathcal{P}_{k} /$ $\sum_{\tilde{k}} \mathcal{P}(m \mid \tilde{k}) \mathcal{P}_{\tilde{k}}$ relates the posterior probability $\mathcal{P}(k \mid m)$ of a hypothesis $k$ (after observing an event $m$ ) to the prior probability $\mathcal{P}_{k}$ of this hypothesis and the conditional probability $\mathcal{P}(k \mid m)$ of the event $m$. The hypotheses $k$ should form a complete and mutually exclusive set.

4. Strictly speaking, the QPT language may not be applicable to the uncollapsing experiment, because it requires linearity of the quantum operation, while after selection of particular realisations and state normalisation the quantum operation is not necessarily linear. However, the linearity is preserved when the selection probability does not depend on the initial state, which is exactly the case for a perfect uncollapsing - see Equation (32). It is also possible to show that even in the imperfect non-linear case the uncollapsing fidelity defined via the 'naive' QPT language practically coincides with the rigorous definition via the average state fidelity [23].

5. We use normalisation of the shot noise, in which $S_{I}=2 e I(1-\mathcal{T})$, where $\mathcal{T}$ is the QPC transparency.

6. In this example, Plato could change his uncollapsing strategy to simply apply a tailored unitary to shift the disturbed state $\left|\psi_{1, m}\right\rangle$ back to its original state $\left|\psi_{1}\right\rangle$. Under this modified strategy, the success probability will be $\mathcal{P}$, which may or may not exceed $P_{S}$, depending on the strength of the measurement. However, in this case,
Plato himself will not know whether the strategy succeeded or not, and therefore cannot claim to have an uncollapse procedure.

7. It is easy to generalise Equations (44)-(47) to include the detector non-ideality and cross-correlation between the output and back-action noises (as in [13]). Non-ideality leads to the extra dephasing term $-\gamma_{d} \sigma_{12}$ in Equation (47) for $\dot{\sigma}_{12}$, while the cross-correlation brings the term $i K\left[I(t)-I_{0}\right] \sigma_{12}$ into the same equation. The formulation of Equations (44)-(47) corresponds to the approach developed in [39].

\section{Notes on contributors}

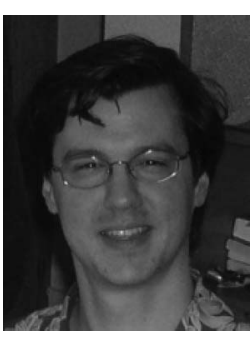

Andrew Jordan received his Ph.D. in Physics from University of California, Santa Barbara in 2002. He subsequently worked at the University of Geneva, Switzerland and Texas A\&M University, and in 2006 he joined the faculty of the University of Rochester (NY), where he remains professor of physics. Interests include mesoscopic physics and foundational topics in quantum mechanics, measurement theory in particular. He has recently been working to develop applications of weak values to precision measurements in optics.

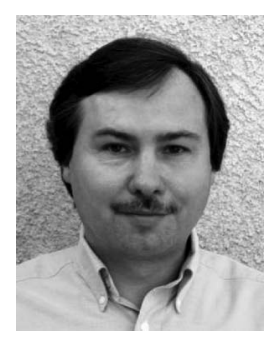

Alexander Korotkov obtained his M.S. (1986) and Ph.D. (1991) degrees in Physics from the Moscow State University. He worked at the Moscow State University, the State University of New York at Stony Brook, and since 2000 he joined the faculty of the University of California, Riverside, where at present he is Professor of Electrical Engineering. In the 1990s he was mainly working on problems in Coulomb blockade and single-electron devices. He was always interested in the controversy of the wavefunction collapse, and since 1998 has been developing the theory of non-projective quantum measurement of solidstate qubits.

\section{References}

[1] J. von Neumann, Mathematical Foundations of Quantum Mechanics, Princeton University Press, Princeton, 1955.

[2] J.A. Wheeler, Law without law, in Quantum Theory and Measurement, J.A. Wheeler and W.H. Zurek, eds., Princeton Series in Physics, Princeton University Press, Princeton, 1983.

[3] E.B. Davies, Quantum Theory of Open Systems, Academic, London, 1976; K. Kraus, States, Effects, and Operations: Fundamental Notions of Quantum Theory, Springer, Berlin, 1983; S. Holevo, Statistical Structure of Quantum Theory, Springer, Berlin, 2001.

[4] M.A. Nielsen and I.L. Chuang, Quantum Computation and Quantum Information, Cambridge University Press, Cambridge, 2000. See Secs. 2.2.3 and 2.4.1 for quantum measurement. J. Preskill, Lecture Notes for Physics 229: Quantum Information and Computation. Available at http://theory.caltech.edu/people/preskill, see Ch. 3 for quantum measurement.

[5] M.B. Mensky, Decoherence and the theory of continuous quantum measurements, Phys. Usp. 41 (1998), p. 923. 
[6] C.M. Caves, Quantum mechanics of measurements distributed in time. A path-integral formulation, Phys. Rev. D 33 (1986), p. 1643; Quantum mechanics of measurements distributed in time. II. Connections among formulations, 35 (1987), p. 1815.

[7] M.B. Plenio and P.L. Knight, The quantum-jump approach to dissipative dynamics in quantum optics, Rev. Mod. Phys. 70 (1998), p. 101; T. Erber and S.J. Putterman, Quantum jumps in a single atom; prolonged darkness in the fluorescence of a resonantly driven cascade, Phys. Lett. A 141 (1989), p. 43.

[8] H.J. Carmichael, An Open System Approach to Quantum Optics, Lecture Notes in Physics, Springer, Berlin, 1993; A.D.F. Walls and G.J. Milburn, Quantum Optics, Springer, Berlin, 2006; H.M. Wiseman and G.J. Milburn, Quantum theory of field-quadrature measurements, Phys. Rev. A 47 (1993), p. 642.

[9] N. Gisin and I.C. Percival, The quantum-state diffusion model applied to open systems, J. Phys. A 25 (1992), p. 5677.

[10] J. Dalibard, Y. Castin, and K. Mølmer, Wave-function approach to dissipative processes in quantum optics, Phys. Rev. Lett. 68 (1992), p. 580.

[11] Y. Aharonov, D.Z. Albert, and L. Vaidman, How the result of a measurement of a component of the spin of a spin-1/2 particle can turn out to be 100, Phys. Rev. Lett. 60 (1988), p. 1351.

[12] V.P. Belavkin, Quantum stochastic calculus and quantum nonlinear filtering, J. Multivar. Anal. 42 (1992), p. 171.

[13] A.N. Korotkov, Continuous quantum measurement of a double dot, Phys. Rev. B 60 (1999), p. 5737; Selective quantum evolution of a qubit state due to continuous measurement, 63 (2001), p. 115403; A.N. Korotkov, Noisy quantum measurement of solid-state qubits: Bayesian approach, preprint (2002), submitted for publication. Available at arXiv:cond-mat/0209629.

[14] A.N. Korotkov and A.N. Jordan, Undoing a weak quantum measurement of a solid-state qubit, Phys. Rev. Lett. 97 (2006). 166805.

[15] A.N. Jordan and A.N. Korotkov, Uncollapsing the wavefunction, in Coherence and Quantum Optics IX, N.P. Bigelow J.H. Eberly, and C.R. Stroud Jr., eds., Optical Society of America, Washington, DC, 2008, p. 191.

[16] M. Koashi and M. Ueda, Reversing measurement and probabilistic quantum error correction, Phys. Rev. Lett. 82 (1999), p. 2598.

[17] A. Ban, Probabilistically reversible measurements, J. Phys. A 34 (2001), p. 9669; G.M. D'Ariano, On the Heisenberg principle, namely on the information-disturbance trade-off in a quantum measurement, Fortschr. Phys. 51 (2003), p. 318; M.A. Nielsen and C.M. Caves, Reversible quantum operations and their application to teleportation, Phys. Rev. A 55 (1997), p. 2547; M. Ueda, N. Imoto, and $\mathrm{H}$. Nagaoka, Logical reversibility in quantum measurement: General theory and specific examples, Phys. Rev. A 53 (1996), p. 3808; H. Mabuchi and P. Zoller, Inversion of quantum jumps in quantum optical systems under continuous observation, Phys. Rev. Lett. 76 (1996), p. 3108; F. Buscemi, M. Hayashi, M. Horodecki, Global information balance in quantum measurements, Phys. Rev. Lett. 100 (2008), 210504.

[18] For various perspectives on these developments, see A. Gefter, Hello kitty!, New Scientist 194 (2007), p. 32; Z. Merali, Reincarnation can save Schrödinger's cat, Nature (London) 454 (2008), p. 8; C. Bruder and D. Loss, Undoing a quantum measurement, Physics 1 (2008), p. 34.
[19] M.O. Scully and K. Drühl, Quantum eraser: A proposed photon correlation experiment concerning observation and "delayed choice" in quantum mechanics, Phys. Rev. A 25 (1982), p. 2208.

[20] A. Peres, Quantum Theory: Concepts and Methods, Kluwer Academic Publishers, Dordrecht, 1995, p. 373.

[21] N. Katz, M. Neeley, M. Ansmann, R.C. Bialczak, M. Hofheinz, E. Lucero, A. O'Connell, H. Wang, A.N. Cleland, J.M. Martinis, and A.N. Korotkov, Reversal of the weak measurement of a quantum state in a superconducting phase qubit, Phys. Rev. Lett. 101 (2008), 200401.

[22] Y.-S. Kim, Y.-W. Cho, Y.-S. Ra, and Y.-H. Kim, Reversing the weak quantum measurement for a photonic qubit, Opt. Express 17 (2009), p. 11978.

[23] A.N. Korotkov and K. Keane, Decoherence suppression by uncollapsing, preprint (2009). Available at arXiv: 0908.1134

[24] J.M. Martinis, S. Nam, J. Aumentado, and C. Urbina, Rabi oscillations in a large Josephson-junction qubit, Phys. Rev. Lett. 89 (2002), p. 117901; K.B. Cooper, M. Steffen, R. McDermott, R.W. Simmonds, S. Oh, D.A. Hite, D.P. Pappas, and J.M. Martinis, Observation of quantum oscillations between a Josephson phase qubit and a microscopic resonator using fast readout, Phys. Rev. Lett. 93 (2004), 180401; J.M. Martinis, Superconducting phase qubits, Quantum Inf. Process. 8 (2009), p. 81.

[25] N. Katz, M. Ansmann, Radoslaw C. Bialczak, E. Lucero, R. McDermott, M. Neeley, M. Steffen, E.M. Weig, A.N. Cleland, J.M. Martinis, and A.N. Korotkov, Coherent state evolution in a superconducting qubit from partial-collapse measurement, Science 312 (2006), p. 1498.

[26] W. Feller, An Introduction to Probability Theory and Its Applications, Vol. 1, Wiley, New York, 1968.

[27] E.T. Jaynes, Probability Theory: The Logic of Science, Cambridge University Press, Cambridge, 2003.

[28] L.P. Pryadko and A.N. Korotkov, Coherence of a Josephson phase qubit under partial-collapse measurement, Phys. Rev. B 76 (2007), p. 100503(R).

[29] S.A. Gurvitz, Measurements with a noninvasive detector and dephasing mechanism, Phys. Rev. B 56 (1997), 15215; A.N. Korotkov and D.V. Averin, Continuous weak measurement of quantum coherent oscillations, Phys. Rev. B 64 (2001), 165310; H.S. Goan and G.J. Milburn, Dynamics of a mesoscopic charge quantum bit under continuous quantum measurement, Phys. Rev. B 64 (2001), 235307; S. Pilgram and M. Büttiker, Efficiency of mesoscopic detectors, Phys. Rev. Lett. 89 (2002), 200401; A.A. Clerk, S.M. Girvin, and A.D. Stone, Quantum-limited measurement and information in mesoscopic detectors, Phys. Rev. B 67 (2003), 165324; D.V. Averin, Continuous weak measurement of the macroscopic quantum coherent oscillation, in Exploring the Quantum-Classical Frontier: Recent Advances in Macroscopic and Mesoscopic Quantum Phenomena, J.R. Friedman and S. Han, eds., Nova Science, Huntington, NY, 2003, preprint (2000). Available at arXiv:cond-mat 0004364; A. Shnirman, D. Mozyrsky, and I. Martin, Output spectrum of a measuring device at arbitrary voltage and temperature, Europhys. Lett. 67 (2004), p. 840; A.A. Clerk and A.D. Stone, Noise and measurement efficiency of a partially coherent mesoscopic detector, Phys. Rev. B 69 (2004), 245303; A.N. 
Jordan and M. Büttiker, Continuous quantum measurement with independent detector cross correlations, Phys. Rev. Lett. 95 (2005), 220401; N.P. Oxtoby, P. Warszawski, H.M. Wiseman, H.B. Sun, R.E.S. Polkinghorne, Quantum trajectories for the realistic measurement of a solid-state charge qubit, Phys Rev B 71 (2005), 165317; A.A. Clerk, M. H. Devoret, S.M. Girvin, F. Marquardt, and R.J. Schoelkopf, Introduction to quantum noise, measurement and amplification, preprint (2008). Available at arXiv:0810.4729v1.

[30] T. Hayashi, T. Fujisawa, H.-Du. Cheong, Y.-Ha. Jeong, and Y. Hirayama, Coherent manipulation of electronic states in a double quantum dot, Phys. Rev. Lett. 91 (2003), 226804; J.R. Petta, A.C. Johnson, C.M. Marcus, M.P. Hanson, and A.C. Gossard, Manipulation of a single charge in a double quantum dot, Phys. Rev. Lett. 93 (2004), 186802; J.R. Petta, A.C. Johnson, J.M. Taylor, E.A. Laird, A. Yacoby, M.D. Lukin, C.M. Marcus, M.P. Hanson, and A.C. Gossard, Coherent manipulation of coupled electron spins in semiconductor quantum dots, Science 309 (2005), p. 2180; J.M. Elzerman, R. Hanson, J.S. Greidanus, L.H. Willems van Beveren, S. De Franceschi, L.M.K. Vandersypen, S. Tarucha, and L.P. Kouwenhoven, Few-electron quantum dot circuit with integrated charge read out, Phys. Rev. B 67 (2003), 161308(R); A.K. Hüttel, S. Ludwig, K. Eberl, J.P. Kotthaus, Direct control of the tunnel splitting in a one-electron double quantum dot, Phys. Rev. B 72 (2005), R081310; J. Gorman, E.G. Emiroglu, D.G. Hasko, and D.A. Williams, Charge-qubit operation of an isolated double quantum dot, Phys. Rev. Lett. 95 (2005), 090502.

[31] A.N. Jordan and M. Büttiker, Quantum nondemolition measurement of a kicked qubit, Phys. Rev. B 71 (2005). 125333; A.N. Jordan, A.N. Korotkov, and M. Büttiker, Leggett-Garg inequality with a kicked quantum pump, Phys. Rev. Lett. 97 (2006), 026805; N.S. Williams and A.N. Jordan, Weak values and the Leggett-Garg inequality in solid-state qubits, Phys. Rev. Lett. 100 (2008), 026804.

[32] A.N. Jordan and A.N. Korotkov, Qubit feedback and control with kicked quantum nondemolition measurements: A quantum Bayesian analysis, Phys. Rev. B 74 (2006), 085307.

[33] A. Shnirman and G. Schön, Quantum measurements performed with a single-electron transistor, Phys. Rev. B 57 (1998), p. 15400.

[34] S. Redner, A Guide to First-Passage Processes, Cambridge University Press, New York, 2001.

[35] C.W. Gardiner and P. Zoller, Quantum Noise, Springer, Berlin, 2004, Chapter 2.2.3.

[36] R. Ruskov, A. Mizel, and A.N. Korotkov, Crossover of phase qubit dynamics in the presence of a negativeresult weak measurement, Phys. Rev. B 75 (2007), 220501(R).

[37] A. Royer, Reversible quantum measurements on a spin 1/ 2 and measuring the state of a single system, Phys. Rev. Lett. 73 (1994), p. 913; 74 (1995), 1040(E).

[38] B. Øksendal, Stochastic Differential Equations, Springer, Berlin, 1998.

[39] H. Wei and Yu.V. Nazarov, Statistics of measurement of noncommuting quantum variables: Monitoring and purification of a qubit, Phys. Rev. B 78 (2008), 045308 .
[40] A.N. Korotkov, Continuous measurement of entangled qubits, Phys. Rev A 65 (2002). 052304.

[41] A.N. Jordan, B. Trauzettel, and G. Burkard, Weakmeasurement theory of quantum-dot spin qubits, Phys. Rev. B 76 (2007), 155324.

[42] F.H.L. Koppens, C. Buizert, K.J. Tielrooij, I.T. Vink, K.C. Nowack, T. Meunier, L.P. Kouwenhoven, and L.M.K. Vandersypen, Driven coherent oscillations of a single electron spin in a quantum dot, Nature 442 (2006), p. 766.

[43] A.C. Johnson, J.R. Petta, J.M. Taylor, A. Yacoby, M.D. Lukin, C.M. Marcus, M.P. Hanson, and A.C. Gossard, Triplet-singlet spin relaxation via nuclei in a double quantum dot, Nature 435 (2005), p. 925; F.H.L. Koppens, J.A. Folk, J.M. Elzerman, R. Hanson, L.H. Willems van Beveren, I.T. Vink, H.P. Tranitz, W. Wegscheider, L.P. Kouwenhoven, and L.M.K. Vandersypen, Control and detection of singlet-triplet mixing in a random nuclear field, Science 309 (2005), p. 1346.

[44] R. Hanson, L.H. Willems van Beveren, I.T. Vink, J.M. Elzerman, W.J.M. Naber, F.H.L. Koppens, L.P. Kouwenhoven, and L.M.K. Vandersypen, Single-shot readout of electron spin states in a quantum dot using spin-dependent tunnel rates, Phys. Rev. Lett. 94 (2005), 196802.

[45] J. Elzerman, R. Hanson, L.H. Willems van Beveren, B. Witkamp, L.M.K. Vandersypen, L.P. and Kouwenhoven, Single-shot read-out of an individual electron spin in a quantum dot, Nature 430 (2004), p. 431.

[46] K. Ono, D.G. Austing, Y. Tokura, and S. Tarucha, Current rectification by Pauli exclusion in a weakly coupled double quantum dot system, Science 297 (2002), p. 1313; A.C. Johnson, J.R. Petta, C.M. Marcus, M.P. Hanson, and A.C. Gossard, Singlet-triplet spin blockade and charge sensing in a few-electron double quantum dot, Phys. Rev. B 72 (2005), 165308.

[47] A.A. Houck, J. Koch, M.H. Devoret, S.M. Girvin, and R.J. Schoelkoph, Life after charge noise: recent results with transmon qubits, Quantum Inf. Process. 8 (2009), p. 105.

[48] N.S. Williams and A.N. Jordan, Entanglement genesis under continuous parity measurement, Phys. Rev. A 78 (2008), 062322.

\section{Appendix 1: Application of first passage theory to the uncollapse time statistics}

In this Appendix, using the methods of first passage theory [34], we rederive Equation (15) for the uncollapse success probability for the unevolving charge qubit, and also obtain the distribution of the waiting time for the uncollapse strategy of Section 4.2. This method has recently been used to investigate entanglement dynamics of jointly measured qubits [48].

It is convenient to scale time in units of the measurement time, $\tau \equiv t / T M$; then the probability distributions (8) take the simple form

$$
P_{1,2}(r, \tau)=\left(\frac{1}{2 \pi \tau}\right)^{1 / 2} \exp \left(-\frac{(r \mp \tau)^{2}}{2 \tau}\right),
$$

after changing variables from $\bar{I}$ to $r$. These are the solutions of two different classical random walks with dimensionless 
drift velocity $\tilde{v}_{1,2}= \pm 1$ and dimensionless diffusion coefficient $\tilde{D}=1 / 2$ described by the Fokker-Planck equations,

$$
\partial_{\tau} P_{i}(r, \tau)=-\tilde{v}_{i} \partial_{r} P_{i}+\tilde{D} \partial_{r}^{2} P_{i}
$$

In order to solve the first passage problem, we solve first for the Green functions $G(r, \tau)$ of the above equations starting from the initial condition $r=r_{0}$. The solutions from the different drift velocities will be weighted with probabilities $\tilde{p}_{1,2}$. These Green function equations are supplemented with an absorbing boundary condition at the origin $(r=0)$,

$$
G_{i}(r=0, \tau)=0,
$$

in order to account for the statistics of random walks that cross this point at least once. Let us again start by assuming $r_{0}>0$ and consider the other case later. The solution of Equation (65) subject to the condition (66) is most easily found by guessing:

$$
\begin{aligned}
G_{i}(r, \tau)= & \frac{1}{(4 \pi \tilde{D} \tau)^{1 / 2}}\left(\exp \left[-\frac{\left(r-r_{0}-\tilde{v}_{i} \tau\right)^{2}}{4 \tilde{D} \tau}\right]\right. \\
& \left.-\exp \left[-\tilde{v}_{i} r_{0} / \tilde{D}\right] \exp \left[-\frac{\left(r+r_{0}-\tilde{v}_{i} \tau\right)^{2}}{4 \tilde{D} \tau}\right]\right) .
\end{aligned}
$$

In the form written above, it is obvious that the solution obeys the equation of motion (65) and has the correct initial condition $r_{0}$ at $\tau=0$ (because the absorbing boundary condition only permits $r \geq 0$ solutions). Further inspection of the solution is facilitated by factoring out the free Green function, $G_{\text {free }}(r, \tau)=\exp \left[-\left(r-r_{0}-\tilde{v_{i} \tau}\right)^{2} /(4 \tilde{D} \tau)\right] / 4 \pi^{1 / 2}$ to write the solution as

$$
G_{i}(r, \tau)=G_{\text {free }}(r, \tau)\left[1-\exp \left(-\frac{r r_{0}}{\tilde{D} \tau}\right)\right] .
$$

One can now explicitly see that the absorbing boundary condition (66) is satisfied, and the solution is completely positive (as it must be to represent a probability density).

To calculate the first passage time distribution, we first note that the total survival probability that the random walker will be in the interval $r \varepsilon(0, \infty)$ at time $\tau$ is given by $P_{\text {sur }}(\tau)=\int_{0}^{\infty} \mathrm{d} r G(r, \tau)$. However, the only place for the particle to be lost from the system is at the origin. Therefore, the first passage time distribution $P_{\mathrm{fpt}}^{(i)}$ is given by

$$
P_{\mathrm{fpt}}^{(i)}=-\partial_{\tau} P_{\mathrm{sur}}=-\int_{0}^{\infty} \mathrm{d} r \partial_{t} G_{i}(r, \tau)
$$

The next step is to note that the Fokker-Planck equation (65) may be rewritten as a continuity equation, $\partial_{\tau} G_{i}+\partial_{r} J_{i}=0$. This simply means that locally, probability is conserved. The probability current in the continuity equation is $\mathrm{J}_{\mathrm{i}}=$ $-\tilde{D} \partial_{r} G_{i}+\tilde{v}_{i} G_{\text {i }}$ from (65). Substituting this into (69) we find the general result

$$
P_{\mathrm{fpt}}^{(i)}(\tau)=\int_{0}^{\infty} \mathrm{d} r \partial_{r} J_{i}=J_{i}(\infty)-J_{i}(0)=-J_{i}(0)
$$

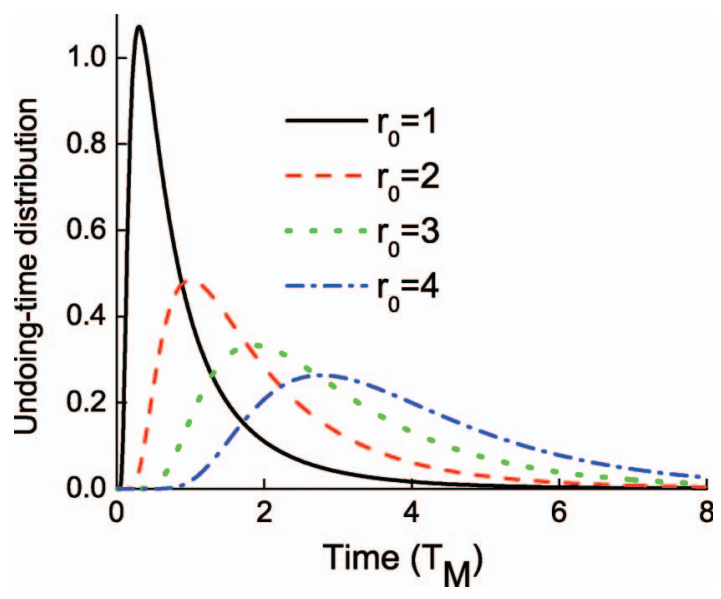

Figure 4. Probability distribution of the time required to undo the measurement. Different plots are for different values of $r_{0}$

because the probability current at infinity vanishes. Applied to our problem, we find

$$
P_{\mathrm{fpt}}^{(i)}(\tau)=\frac{r_{0}}{\left(4 \pi \tilde{D} \tau^{3}\right)^{1 / 2}} \exp \left[-\left(r_{0}+\tilde{v}_{i} \tau\right)^{2} /(4 \tilde{D} \tau)\right] .
$$

The probability $P_{\mathrm{C}}$ that the point $r=0$ is ever crossed is found by integrating (71) over all positive time to obtain

$$
P_{\mathrm{C}}= \begin{cases}\exp \left(-\tilde{v}_{1} r_{0} / \tilde{D}\right)=\exp \left(-2 r_{0}\right), & i=1, \\ 1, & i=2 .\end{cases}
$$

This result may be understood intuitively because if the state is in $i=2$ then the drift $\tilde{v}_{2}=-1$ causes $r(t)$ to evolve from $r_{0}$ to $-\infty$ and therefore must cross 0 at some time, while if the system is in state $i=1$ then the drift $\tilde{v}_{1}=1$ causes $r(t)$ to evolve from $r_{0}$ to $+\infty$. Therefore, in order to cross $r=0$, the noise term must fight against the drift, causing a successful crossing only occasionally.

In order to obtain the normalised first passage distribution (conditioned on crossing), we divide (71) by the probabilities (72) to obtain

$$
P_{\mathrm{fpt}}^{(i)}(\tau \mid C)=\frac{r_{0}}{\left(4 \pi \tilde{D} \tau^{3}\right)^{1 / 2}} \exp \left[-\left(r_{0}-\left|\tilde{v}_{i}\right| \tau\right)^{2} /(4 \tilde{D} \tau)\right]
$$

The mean first passage time may also be calculated from (73) to obtain $\tau_{c, i}=r_{0} /\left|\tilde{v}_{i}\right|=\mathrm{r}_{0}$.

Obtaining analogous results for $r_{0}<0$ is straightforward because the Green function for the Fokker-Planck equation (65) is invariant under the transformation $\left\{r \rightarrow-r, r_{0} \rightarrow-\mathrm{r}_{0}\right.$, $\tilde{v}_{i} \rightarrow-\tilde{v}_{i}$ (or $1 \leftrightarrow 2$ ) $\}$ which is also reflection symmetry about the origin. Therefore, results (71), (72) and (73) can be extended using this symmetry. Combining results, we can now calculate the total uncollapsing probability, $P_{\mathrm{s}}=\tilde{p}_{1} P_{C, 1}+$ $\tilde{p}_{2} P_{C, 2}$ to obtain the result (15) in this new, more powerful way.

In addition to the probability of success, the complete solution of the first-passage problem given above now allows 
us to specify further information about the uncollapsing process. In particular, an important question for an experimental implementation of this idea is how long it is necessary to wait.

Since the distribution of the first passage time (73) does not depend on the bit state, it directly gives the distribution of the waiting time to uncollapse any qubit state. Therefore, rescaling back the time axis in Equation (73), we find the waiting time distribution is

$$
P_{\text {wait }}(t)=\frac{\left|r_{0}\right|}{\left(2 \pi t^{3} / T_{M}\right)^{1 / 2}} \exp \left[\frac{-\left(\left|r_{0}\right|-t / T_{M}\right)^{2}}{2 t / T_{M}}\right]
$$

This distribution is normalised, since we consider only successful attempts of uncollapsing. The fact that the distribution is independent of the initial qubit state is not surprising, since otherwise a successful uncollapsing instance would give us an information about the qubit state (see discussion in Section 5.3).
Using the distribution (74), we can find the mean waiting time to uncollapse

$$
T_{\text {wait }}=T_{M}\left|r_{0}\right|
$$

the standard deviation

$$
\Delta T_{\text {wait }}=T_{M}\left(\left|r_{0}\right|\right)^{1 / 2},
$$

and the most likely waiting time (which maximises $P_{\text {wait }}$ )

$$
T_{l}=T_{M}\left(\left(r_{0}^{2}+9 / 4\right)^{1 / 2}-3 / 2\right) .
$$

The distribution (74) of the waiting time is plotted in Figure 4 for several values of $r_{0}$. Note that it has a long tail, which makes the average value $T_{\text {wait }}$ to be longer than the most likely value $T_{l}$. 